\title{
Electron-impact excitation of $X^{1} \Sigma_{g}^{+}\left(v^{\prime \prime}=0\right)$ to the $a^{\prime \prime}{ }^{1} \Sigma_{g}^{+}, b^{1} \Pi_{u}, c_{3}{ }^{1} \Pi_{u}, o_{3}{ }^{1} \Pi_{u}, b^{\prime}{ }^{1} \Sigma_{u}^{+}, c_{4}^{\prime}{ }^{1} \Sigma_{u}^{+}$, $G^{3} \Pi_{u}$, and $F^{3} \Pi_{u}$ states of molecular nitrogen
}

\author{
M. A. Khakoo, ${ }^{1}$ C. P. Malone, ${ }^{2}$ P. V. Johnson, ${ }^{2}$ B. R. Lewis, ${ }^{3}$ R. Laher, ${ }^{4}$ S. Wang, ${ }^{1}$ V. Swaminathan, ${ }^{5}$ \\ D. Nuyujukian, ${ }^{5}$ and I. Kanik ${ }^{2}$ \\ ${ }^{1}$ Department of Physics, California State University, Fullerton, California 92834, USA \\ ${ }^{2}$ Jet Propulsion Laboratory, California Institute of Technology, 4800 Oak Grove Drive, Pasadena, California 91109, USA \\ ${ }^{3}$ Research School of Physical Sciences and Engineering, The Australian National University, Canberra ACT 0200, Australia \\ ${ }^{4}$ Spitzer Science Center, California Institute of Technology, Pasadena, California 91125, USA \\ ${ }^{5}$ Troy High School, Dorothy Lane, Fullerton, California 92831, USA
}

(Received 24 September 2007; published 7 January 2008)

\begin{abstract}
Measurements of differential cross sections (DCSs) for electron-impact excitation of the $a^{\prime \prime}{ }^{1} \Sigma_{g}^{+}, b^{1} \Pi_{u}$, $c_{3}{ }^{1} \Pi_{u}, o_{3}{ }^{1} \Pi_{u}, b^{\prime}{ }^{1} \Sigma_{u}^{+}, c_{4}^{\prime}{ }^{1} \Sigma_{u}^{+}, G^{3} \Pi_{u}$, and $F^{3} \Pi_{u}$ states in $\mathrm{N}_{2}$ from the $X^{1} \Sigma_{g}^{+}\left(v^{\prime \prime}=0\right)$ ground level are presented. The DCSs were obtained from energy-loss spectra in the region of 12 to $13.82 \mathrm{eV}$ measured at incident energies of $17.5,20,30,50$, and $100 \mathrm{eV}$ and for scattering angles ranging from $2^{\circ}$ to $130^{\circ}$. The analysis of the spectra follows a different algorithm from that employed in a previous study of $\mathrm{N}_{2}$ for the valence states [Khakoo et al. Phys. Rev. A 71, 062703 (2005)], since the ${ }^{1} \Pi_{u}$ and ${ }^{1} \Sigma_{u}^{+}$states form strongly interacting Rydberg-valence series. The results are compared with existing data.
\end{abstract}

DOI: $10.1103 /$ PhysRevA.77.012704

PACS number(s): $34.80 . \mathrm{Gs}, 34.50 . \mathrm{Bw}$

\section{INTRODUCTION}

Electron-impact excitation of atomic and molecular targets plays a key role in a host of natural (auroras, dayglow, interstellar emissions) and man-made environments (plasmas in lasers, etching plasmas, lighting discharges, plasma sterilization, etc.). Considerable effort has been made in the past to both theoretically and experimentally determine electron scattering cross sections for these targets. Whereas significant progress has been made for the excitation of atomic targets, molecular targets remain difficult to model on account of their reduced symmetry and the inclusion of nuclear rotational and vibrational degrees of freedom, which couple to the electronic motion.

Recently, we carried out a careful and extensive series of energy-loss measurements in molecular nitrogen covering the energy-loss range of $6.25-11.25 \mathrm{eV}$ from which we determined differential cross sections (DCSs) for excitation of the $A^{3} \Sigma_{u}^{+}, B{ }^{3} \Pi_{g}, W^{3} \Delta_{u}, B^{\prime}{ }^{3} \Sigma_{u}^{-}, a^{\prime}{ }^{1} \Sigma_{u}^{-}, a^{1} \Pi_{g}, w^{1} \Delta_{u}$, and $C^{3} \Pi_{u}$ electronic levels from the $X^{1} \Sigma_{g}^{+}\left(v^{\prime \prime}=0\right)$ ground state [1]. Whereas, in some cases, significant disagreements were observed between our results and those of previous measurements, very recent $a b$ initio theoretical studies by da Costa and Lima $[2,3]$ using a perturbative multichannel Schwinger method and Tashiro and Morokuma [4] using a closecoupling $R$-matrix method show significantly improved agreement with our results. We note here that better agreement was obtained between our earlier DCSs [1] and the close-coupling code [4], though improving convergence was evident in [3]. Ongoing accurate time-of-flight measurements by Buckman [5] are expected to shed more definitive light in this area. Here, we have extended our $\mathrm{N}_{2}$ measurements to examine excitation features in the energy-loss range of approximately $12-13.82 \mathrm{eV}$, which includes the $a^{\prime \prime}{ }^{1} \Sigma_{g}^{+}, b{ }^{1} \Pi_{u}$, $c_{3}{ }^{1} \Pi_{u}, o_{3}{ }^{1} \Pi_{u}, b^{\prime}{ }^{1} \Sigma_{u}^{+}, c_{4}^{\prime}{ }^{1} \Sigma_{u}^{+}, G^{3} \Pi_{u}$, and $F^{3} \Pi_{u}$ states, with the ungerade potential-energy curves shown in Fig. 1. Electron-impact excitation of the $b{ }^{1} \Pi_{u}, c_{3}{ }^{1} \Pi_{u}, o_{3}{ }^{1} \Pi_{u}$, $b^{\prime}{ }^{1} \Sigma_{u}^{+}$, and $c_{4}^{\prime}{ }^{1} \Sigma_{u}^{+}$states are especially important because they are dipole-coupled to the ground $X^{1} \Sigma_{g}^{+}$state, radiate copiously, and therefore dominate the observable extreme ultraviolet spectrum of $\mathrm{N}_{2}$ plasmas.

The spectroscopy of these latter states is well known to be complicated by very strong Rydberg-valence, RydbergRydberg, and valence-valence interactions within the ${ }^{1} \Pi_{u}$ and ${ }^{1} \Sigma_{u}^{+}$manifolds [6-8], and similar interactions are expected within the ${ }^{3} \Pi_{u}$ manifold [9], which is responsible for the singlet-state predissociation [10]. As has been pointed out

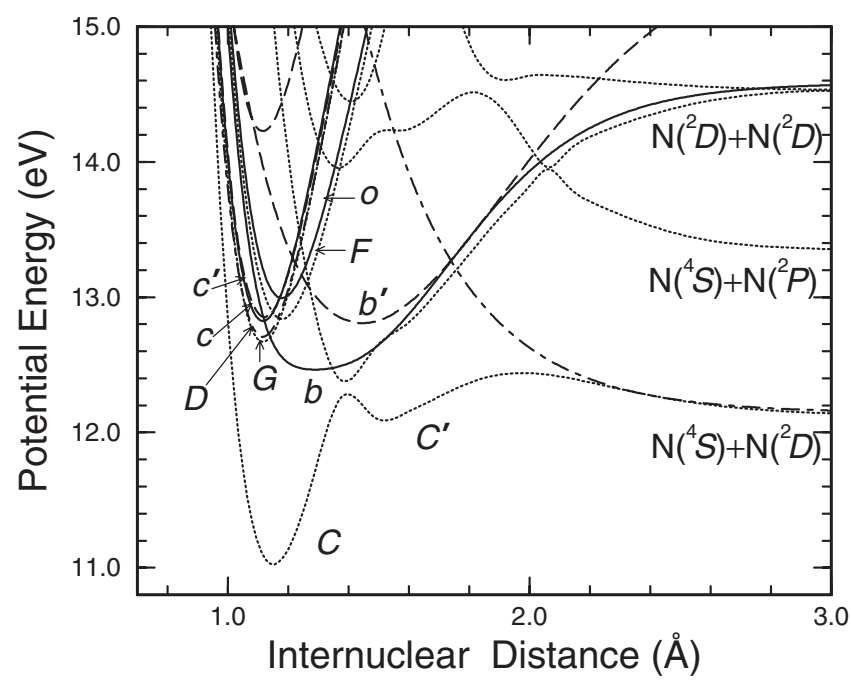

FIG. 1. Representative potential-energy curves for excited, ungerade electronic states of $\mathrm{N}_{2}$ in the region of interest to this work, with the energy scale referenced to the minimum in the ground $X^{1} \Sigma_{g}^{+}\left(v^{\prime \prime}=0\right)$ state potential (not shown). The states studied here are labeled: ${ }^{1} \Pi_{u}$ states, solid line; ${ }^{1} \Sigma_{u}^{+}$states, dashed line; ${ }^{3} \Pi_{u}$ states, dotted line; ${ }^{3} \Sigma_{u}^{+}$states, dot-dashed line. Note that the potentials are shown in a diabatic (crossing) representation, except in the case of the ${ }^{3} \Pi_{u}$ states, where they are taken from the $a b$ initio calculations of Partridge [66]. 
in a study by Lewis et al. [11] of the effects of such interactions in $\mathrm{O}_{2}$ on the intensities in electron energy-loss spectra, consequent quantum-interference effects lead to intensity anomalies in vibrational series, which vary with the scattering angle and energy. Thus, the invariant Franck-Condon (FC) factor assumption, which is implicit in the techniques normally used in the spectral decomposition of electron energy-loss spectra (including in [1]), is invalid in the present case. This is evident by comparing the diabatic (crossing) and adiabatic (avoided-crossing) potential energy curves given by Stahel et al. [8] for $\mathrm{N}_{2}$ in our region of interest (also see Fig. 1). Thus, the present spectral analysis employs a different technique, in that the spectra are fitted with all vibrational levels of a given electronic transition treated as independent transitions. We note that the exception here is the $a^{\prime \prime}{ }^{1} \Sigma_{g}^{+}$state, which is apparently unaffected by perturbative interactions with nearby states. Therefore, its analysis is simplified, in that the intensities of its vibrationallevel energy-loss features are found to remain proportional to the FC factors for their excitation from the $X^{1} \Sigma_{g}^{+}\left(v^{\prime \prime}=0\right)$ ground state [12], under all scattering conditions (see Sec. III A).

Past investigations of the electron-impact excitation of the states of $\mathrm{N}_{2}$ covered in this work are now summarized below. We note that Wilden et al. [13] measured electron energyloss spectra between 5 and $25 \mathrm{eV}$ for various impact energies and scattering angles, but did not derive DCSs for the states investigated in the present work. The reader is also referred to the reviews of Itikawa et al. [14] and Itikawa [15], which cover research on $e^{-}+\mathrm{N}_{2}$ until 2005.

\section{A. The $a^{\prime \prime} \mathbf{\Sigma}_{g}^{+}$state}

In the united-atom orbital designation, the $a^{\prime \prime}{ }^{1} \Sigma_{g}^{+}$state is a $3 s \sigma_{g}$ Rydberg state built on the ground state of $\mathrm{N}_{2}{ }^{+}$. This state can decay to the ground state only by quadrupole radiation, giving rise to the weak Dressler-Lutz system observed in high-pressure plasmas [16]. Electron-impact excitation of this system was studied for only the $X^{1} \Sigma_{g}^{+}\left(v^{\prime \prime}=0\right)$ $\rightarrow a^{\prime \prime}{ }^{1} \Sigma_{g}^{+}\left(v^{\prime}=0\right)$ transition by Lassettre et al. [17], at the high incident energy $\left(E_{0}\right)$ of $500 \mathrm{eV}$ and small electron scattering angles $(\theta)$, in order to determine the generalized oscillator strength (GOS) as a function of momentum transfer squared $\left(K^{2}\right)$, showing that the GOS $\rightarrow 0$ as $K^{2} \rightarrow 0$, typical of a forbidden transition. The first DCS measurements for $X^{1} \Sigma_{g}^{+}\left(v^{\prime \prime}=0\right) \rightarrow a^{\prime \prime}{ }^{1} \Sigma_{g}^{+}\left(v^{\prime}=0,1\right)$ were performed by Cartwright et al. [18], normalized to the then-available elastic $\mathrm{N}_{2}$ DCS standards. Their DCSs were obtained for $E_{0}$ ranging from 15 to $50 \mathrm{eV}$ and $\theta$ from $10^{\circ}$ to $130^{\circ}$, and were later renormalized to improved elastic $\mathrm{N}_{2}$ DCS standards by Trajmar et al. [19]. It must be noted here that the results of [19] are in mixed agreement with those of [1] for the excitation of the $A^{3} \Sigma_{u}^{+}, B{ }^{3} \Pi_{g}, W^{3} \Delta_{u}, B^{\prime}{ }^{3} \Sigma_{u}^{-}, a^{\prime}{ }^{1} \Sigma_{u}^{-}, a^{1} \Pi_{g}$, $w^{1} \Delta_{u}$, and $C^{3} \Pi_{u}$ electronic manifolds. In this work, we will compare directly with the values in [19]. Brunger and Teubner [20] made similar measurements for $E_{0}$ ranging from 15 to $50 \mathrm{eV}$ and $\theta$ from $10^{\circ}$ to $90^{\circ}$ using the best elastic $\mathrm{N}_{2}$ calibration standards available to date. At about the same time, DCS ratios for the electron-impact ex- citation of the $X^{1} \Sigma_{g}^{+}\left(v^{\prime \prime}=0\right) \rightarrow a^{\prime \prime}{ }^{1} \Sigma_{g}^{+}\left(v^{\prime}=0,1\right)$ transition relative to the $X^{1} \Sigma_{g}^{+}\left(v^{\prime \prime}=0\right) \rightarrow\left[b^{1} \Pi_{u}\left(v^{\prime}=0-7\right)+c_{3}{ }^{1} \Pi_{u}\left(v^{\prime}\right.\right.$ $\left.=0,1)+c_{4}^{\prime}{ }^{1} \Sigma_{u}^{+}\left(v^{\prime}=0,1\right)\right]$ transition were measured by Furlan et al. [21] at $E_{0}=35 \mathrm{eV}$, for $\theta$ nominally from $-30^{\circ}$ to $80^{\circ}$. Excellent agreement was found between these data and those obtained from an earlier work by Trajmar et al. [22]. Later, Zubek and King [23] made similar DCS measurements, using the same elastic $\mathrm{N}_{2}$ calibration standards as [19], at $E_{0}$ $=17.5$ and $20 \mathrm{eV}$, for $\theta$ from $10^{\circ}$ to $100^{\circ}$. Their DCSs showed some disagreement with those of Trajmar et al. [19] and Brunger and Teubner [20]. Also, a (small) dip in the DCSs near $\theta=20^{\circ}$, for $E_{0}>20 \mathrm{eV}$, was noted by Zubek and King [23] and by Furlan et al. [21], but not investigated in any detail. DCSs for the electron-impact excitation of the $a^{\prime \prime}{ }^{1} \Sigma_{g}^{+}\left(v^{\prime}=0,1\right)$ state resulting from the present experiments, and in particular an in-depth discussion of the aforementioned dip, were recently reported elsewhere [12].

\section{B. The ${ }^{1} \Pi_{u}$ states}

The $b^{1} \Pi_{u}$ valence and $3 p \pi_{u} c_{3}{ }^{1} \Pi_{u}$ and $3 s \sigma_{g} o_{3}{ }^{1} \Pi_{u}$ Rydberg states of $\mathrm{N}_{2}$ are well known to exhibit strong mutual interactions, with a detailed semiempirical treatment of these coupled states performed in a benchmark paper by Stahel et $a l$. [8], followed by a modern $a b$ initio treatment by Spelsberg and Meyer [7]. Excitation of the $b{ }^{1} \Pi_{u}$ state gives rise to the intense $b^{1} \Pi_{u} \rightarrow X^{1} \Sigma_{g}^{+}$Birge-Hopfield I bands in the extreme ultraviolet (euv) and the $b^{1} \Pi_{u} \rightarrow a^{1} \Pi_{g}$ GaydonHerman near-uv bands. The Birge-Hopfield I bands have been investigated in detail by Carroll and Collins [24] and Dressler [25]. Excitation of the $c_{3}{ }^{1} \Pi_{u}$ and $o_{3}{ }^{1} \Pi_{u}$ states gives rise to the $c_{3}{ }^{1} \Pi_{u} \rightarrow X^{1} \Sigma_{g}^{+}$Worley-Jenkins and the $o_{3}{ }^{1} \Pi_{u} \rightarrow X^{1} \Sigma_{g}^{+}$Worley series of Rydberg bands in the euv region. Differential electron scattering of the $b^{1} \Pi_{u}, c_{3}{ }^{1} \Pi_{u}$, and $o_{3}{ }^{1} \Pi_{u}$ Rydberg-valence states was carried out by Chutjian et al. [26], who unfolded the energy-loss spectrum based on data in the form of excitation energies and FC factors from $[24,25,27,28]$. [Of note, more recent diabatic Rydberg-Klein-Rees- (RKR-) derived FC factors are available for the $c_{3}{ }^{1} \Pi_{u}, o_{3}{ }^{1} \Pi_{u}, b^{\prime}{ }^{1} \Sigma_{u}^{+}$, and $c_{4}^{\prime}{ }^{1} \Sigma_{u}^{+}$states [29].] However, as mentioned above, since it is now known that these FC factors are not constant with $\theta$, due to strong Rydberg-valence interactions, their method of data analysis (i.e., fixed FC factors) was technically flawed. Also, to account for problems in fitting their spectra, they had to allow for the addition of several unknown states-i.e., $M 1, M 2$, and $M 3$ (see Sec. III A 4). Although the DCS data of [26] were corrected later by Trajmar et al. [19], using a better elastic $\mathrm{N}_{2}$ standard, any systematic problems resulting from fixed FC-factor fitting were not addressed. Nevertheless, prior to the present work, the DCSs of Chutjian et al. [26] (renormalized by Trajmar et al. [19]) remained the only measured DCSs available for these coupled ${ }^{1} \Pi_{u}$ states of $\mathrm{N}_{2}$. Note that Ratliff et al. [30] provided an energy-loss spectrum and integral cross sections (ICSs) at 60 and $100 \mathrm{eV}$ incident electron energies for the $b^{1} \Pi_{u}$ state of $\mathrm{N}_{2}$, but provided no DCS results.

\section{The ${ }^{1} \Sigma_{u}^{+}$states}

Just as for the ${ }^{1} \Pi_{u}$ states discussed above, the $b^{\prime}{ }^{1} \Sigma_{u}^{+}$valence and $3 p \sigma_{u} c_{4}^{\prime}{ }^{1} \Sigma_{u}^{+}$Rydberg states of $\mathrm{N}_{2}$ are also strongly 
coupled $[8,10,27]$. Excitation of the $b^{\prime}{ }^{1} \Sigma_{u}^{+}$and $c_{4}^{\prime}{ }^{1} \Sigma_{u}^{+}$states gives rise to the Carroll-Collins-Yoshino and CarrollYoshino bands, respectively, which are both in the euv region. The Rydberg-valence interaction between these states leads to significant mutual perturbations, studied by Dressler [25] and Carroll et al. [31], which show up as irregularities in the corresponding vibrational and rotational structure. Once again, the DCSs of Chutjian et al. [26] are the only measured data available for comparison with the present results for these coupled ${ }^{1} \Sigma_{u}^{+}$states. The most recent theoretical DCSs for the $b^{\prime 1} \Sigma_{u}^{+}$and $c_{4}^{\prime 1} \Sigma_{u}^{+}$states were published by $\mathrm{Mu}-$ Tao and McKoy [32], using a distorted-wave calculation. Prior theoretical efforts are discussed by Mu-Tao and McKoy.

Much work has been done on the spectroscopy of the coupled ${ }^{1} \Pi_{u}$ and ${ }^{1} \Sigma_{u}^{+}$states of $\mathrm{N}_{2}$, with the early studies and historical developments summarized in the review of Lofthus and Krupenie [16]. An understanding of the coupling mechanisms and their consequences-i.e., strong perturbations in both energy and intensity - can be gained from the work of Stahel et al. [8] and Spelsberg and Meyer [7], together with the informative book of Lefebvre-Brion and Field [6]. Recently, there has been a reawakening of interest in the spectroscopy and dissociation dynamics of $\mathrm{N}_{2}$ in the euv region, driven mainly by important applications in the area of planetary-atmospheric modeling. In particular, recent laserbased [9,33-35] and synchrotron-based [36,37] experimental studies, together with the photofragment study of van der Kamp et al. [38], have provided valuable new spectroscopic, intensity, and linewidth information. Of particular relevance here is the convincing reassignment in [38] of the vibrational levels of the $F^{3} \Pi_{u}$ state, which were assumed in the electron-scattering study of Chutjian et al. [26]. These data have enabled progress to be made in establishing the predissociation mechanism for the $b{ }^{1} \Pi_{u}, c_{3}{ }^{1} \Pi_{w}$, and $o_{3}{ }^{1} \Pi_{u}$ states of $\mathrm{N}_{2}$, including a recent coupled-channels study by Lewis $e t$ al. [10] confirming that predissociation is caused indirectly by spin-orbit coupling between the $b^{1} \Pi_{u}$ and $C^{3} \Pi_{u}$ valence states, the latter of which is electrostatically coupled to the $C^{\prime}{ }^{3} \Pi_{u}$ valence-state continuum. Further, a theoretical investigation of the absorption spectrum of $\mathrm{N}_{2}$ above the ionization threshold has been made by Lefebvre-Brion [39], based on the experimental laser-based study of McCormack et al. [40].

The present work is a first attempt to determine the DCSs for the $b{ }^{1} \Pi_{u}, c_{3}{ }^{1} \Pi_{u}, o_{3}{ }^{1} \Pi_{u}, b^{\prime}{ }^{1} \Sigma_{u}^{+}$, and $c_{4}^{\prime}{ }^{1} \Sigma_{u}^{+}$states of $\mathrm{N}_{2}$, in the energy-loss region of $12-13.82 \mathrm{eV}$, using an analysis that allows for the presence of strong interactions between these states, not considered in previous analyses. Further, the present work attempts to account for contributions resulting from excitation of the $D^{3} \Sigma_{u}^{+}, G^{3} \Pi_{u}$, and $F^{3} \Pi_{u}$ states (see Table I) to the energy-loss spectra of the dipole-allowed states and to obtain DCSs for these excitations in an effort to complete the picture in this energy-loss region.

\section{EXPERIMENT}

\section{A. Experimental techniques and procedures}

Detailed descriptions of the experimental apparatus are given in Khakoo et al. [41]. Briefly, cylindrical electrostatic optics and double hemispherical energy selectors were utilized, both in the electron gun and in the detector. Energyloss spectra, including both the elastic peak and the inelastic region of interest, were collected at fixed impact energies and scattering angles by repetitive, multichannel-scaling techniques. The target $\mathrm{N}_{2}$ beam was formed by effusing the gas through a capillary array (with a collimation aspect ratio of $\sim 100: 1)$ driven by a pressure of a few Torr. The incident energy $E_{0}$ of the electron beam was calibrated by setting $E_{0}$ greater than the $\mathrm{N}_{2}$ ionization potential, tuning the spectrometer analyzer to the elastic peak (energy loss $E=0$ ), and then taking an energy-loss spectrum extending beyond the point where $E=E_{0}$. When $E=E_{0}$, there is a distinct cutoff in scattered signal. The incident energy was determined by comparing the nominal $E_{0}$ with the location of the observed cutoff. The correct value of $E_{0}$ could be set to within $50 \mathrm{meV}$ by adjusting the appropriate electron gun bias power supply to obtain the appropriate cutoff voltage. This technique has proven simpler than traditional methods based on the $2^{2} S$ resonance in the electron-helium elastic scattering channel, and yet gave excellent agreement with this method. The energy-loss cutoff method has the advantage of expediency, over the helium resonance method, as one does not have to change gases from $\mathrm{N}_{2}$ to $\mathrm{He}$ during an experiment, although a slight penalty in precision is incurred. The spectrometer was found to be very stable, yielding currents of about 7-10 nA with an energy resolution of approximately $40 \mathrm{meV}$ full width at half maximum (FWHM).

The procedure for obtaining normalized cross sections consisted of several steps.

\section{Minimizing transmission effects}

The spectrometer was tuned in an iterative manner for each $E_{0}$ at $90^{\circ}$, with the background gas signal removed using the moveable source method developed by Hughes and co-workers [42] (see Sec. II A 3). This was performed so that the elastic-to-inelastic ratios closely reproduced those from the time-of-flight (TOF) work of LeClair and Trajmar [43] $\left(\right.$ at $90^{\circ}$ ), which are accurate on a relative scale to $\pm 5 \%$. The analyzer was baked and maintained in a very clean vacuum environment so that this response remained stable. At low residual energy $E_{R}\left(=E_{0}-E\right)$, transmission effects become more prominent. Therefore, a further correction was applied based on the helium ionization continuum at $E_{0}=30 \mathrm{eV}$, which has been shown to be flat to within 5\% [44]. This could be described closely (within 10\%) by the function [1]

$$
T\left(E_{R}\right)=0.055 \ln \left(E_{R}\right)+0.87 .
$$

This function was applied to spectra obtained at $E_{0}$ $=17.5 \mathrm{eV}$ to account for energy-dependent transmission in the spectrometer. At higher energies, $E_{R}>4 \mathrm{eV}$, transmission effects were not significant and no analytical correction was made.

\section{Determining inelastic energy-loss intensities}

Energy-loss spectra were then accumulated in the energyloss range of $12-14.5 \mathrm{eV}$ and unfolded in the energy-loss range of $12-13.82 \mathrm{eV}$, leaving all vibrational levels within 
TABLE I. (a) Energy-loss positions in eV (levels ordered by energy) for the excitation of the $a^{\prime \prime}{ }^{1} \Sigma_{g}^{+}, b^{1} \Pi_{u}, c_{3}{ }^{1} \Pi_{u}, o_{3}{ }^{1} \Pi_{u}, b^{\prime}{ }^{1} \Sigma_{u}^{+}$, $c_{4}^{\prime}{ }^{1} \Sigma_{u}^{+}, D^{3} \Sigma_{u}^{+}, G^{3} \Pi_{u}$, and $F^{3} \Pi_{u}\left(v^{\prime}\right)$ levels from the $X^{1} \Sigma_{g}^{+}\left(v^{\prime \prime}=0\right)$ ground state. References: P, present work, limited to maximum $E$ of $13.82 \mathrm{eV}$ (the $E$ values used in the unfolding of present spectra); SLD, Stahel et al. [8]; H, Hammond et al. [65]; LK, Lofthus and Krupenie [16], either directly from tables or from RKR calculations using their molecular constants for the electronic levels; and T, Trajmar [46] (based on $[19,26,30]$, etc.). Italicized data correspond to pairs of levels that are not resolvable by our measurement and unfolding procedures. (b) Normalized "relative excitation probabilities (REPs)" with vibrational levels grouped by electronic state. Note that the summations of P (renorm) for individual states are the corresponding scaling factors in Table IV. See text for additional discussion.

\begin{tabular}{|c|c|c|c|c|c|c|c|c|c|c|}
\hline \multirow{2}{*}{$\begin{array}{l}\text { (a) } \\
\text { Level }\end{array}$} & \multicolumn{5}{|c|}{ Excitation energies $(\mathrm{eV})$} & \multirow{2}{*}{$\begin{array}{c}(b) \\
\text { Level }\end{array}$} & \multicolumn{4}{|c|}{ Relative excitation probabilities (REPs) } \\
\hline & $\mathrm{P}$ & SLD & $\mathrm{H}$ & LK & $\mathrm{T}$ & & $\mathrm{T}$ & SLD & $\mathrm{P}$ & $\mathrm{P}$ (renorm) \\
\hline$a^{\prime \prime}(0)$ & 12.255 & & 12.255 & & 12.253 & $a^{\prime \prime}(0)$ & 0.870 & & $0.848 \pm 0.031$ & \\
\hline$b(0)$ & 12.4997 & 12.499 & 12.500 & 12.4996 & 12.500 & $a^{\prime \prime}(1)$ & 0.130 & & $0.152 \pm 0.022$ & \\
\hline$a^{\prime \prime}(1)$ & 12.516 & & 12.520 & & 12.516 & & & & & \\
\hline$M ?$ & 12.540 & & & & & $b(0)$ & 0.010 & 0.0101 & $0.015 \pm 0.001$ & $0.014 \pm 0.001$ \\
\hline$b(1)$ & 12.5784 & 12.580 & 12.579 & 12.5796 & 12.575 & $b(1)$ & 0.057 & 0.0459 & $0.053 \pm 0.003$ & $0.051 \pm 0.003$ \\
\hline$b(2)$ & 12.6652 & 12.666 & 12.665 & 12.6671 & 12.663 & $b(2)$ & 0.126 & 0.1169 & $0.127 \pm 0.007$ & $0.123 \pm 0.007$ \\
\hline$b(3)$ & 12.7532 & 12.753 & 12.754 & 12.7598 & 12.750 & $b(3)$ & 0.230 & 0.2190 & $0.221 \pm 0.010$ & $0.215 \pm 0.010$ \\
\hline$G(0)$ & 12.8097 & & 12.814 & & 12.810 & $b(4)$ & 0.360 & 0.3028 & $0.289 \pm 0.023$ & $0.281 \pm 0.022$ \\
\hline$b(4)$ & 12.8384 & 12.838 & 12.839 & 12.8559 & 12.835 & $b(5)$ & 0.018 & 0.0127 & $0.012 \pm 0.008$ & $0.012 \pm 0.008$ \\
\hline$D(0)$ & 12.8414 & & 12.842 & 12.8412 & 12.841 & $b(6)$ & 0.016 & 0.0196 & $0.032 \pm 0.010$ & $0.032 \pm 0.010$ \\
\hline$b^{\prime}(0)$ & 12.8535 & 12.853 & & 12.8574 & 12.861 & $b(7)$ & 0.089 & 0.1112 & $0.092 \pm 0.012$ & $0.090 \pm 0.012$ \\
\hline$c_{3}(0)$ & 12.9115 & 12.913 & 12.914 & 12.920 & 12.910 & $b(8)$ & 0.002 & 0.0079 & $0.005 \pm 0.001$ & $0.005 \pm 0.001$ \\
\hline$c_{4}^{\prime}(0)$ & 12.9344 & 12.935 & 12.937 & 12.9487 & 12.935 & $b(9)$ & 0.017 & 0.0298 & $0.026 \pm 0.016$ & $0.026 \pm 0.015$ \\
\hline$b^{\prime}(1)$ & 12.9463 & 12.946 & & 12.951 & 12.956 & $b(10)$ & 0.050 & 0.0489 & $0.055 \pm 0.007$ & $0.053 \pm 0.007$ \\
\hline$b(5)$ & 12.9811 & 12.981 & 12.981 & 12.9538 & 12.950 & $b(11)$ & 0.017 & 0.0271 & $0.021 \pm 0.001$ & $0.020 \pm 0.001$ \\
\hline$F(0)$ & 12.9849 & & & & 12.980 & $b(12)$ & 0.003 & 0.0094 & $0.015 \pm 0.004$ & $0.014 \pm 0.004$ \\
\hline$b^{\prime}(2)$ & 13.0371 & 13.037 & & 13.0427 & 13.049 & $b(13)$ & 0.001 & 0.0137 & $0.034 \pm 0.009$ & $0.033 \pm 0.008$ \\
\hline$b(6)$ & 13.0612 & 13.062 & 13.061 & 13.0522 & 13.062 & $b(14)$ & 0.003 & 0.0249 & $0.004 \pm 0.004$ & $0.004 \pm 0.004$ \\
\hline$o_{3}(0)$ & 13.103 & 13.103 & 13.103 & 13.103 & 13.100 & & & & & \\
\hline$D(1)$ & 13.1066 & & & 13.112 & 13.097 & $c_{3}(0)$ & 0.761 & 0.4891 & $0.494 \pm 0.063$ & $0.494 \pm 0.063$ \\
\hline$b^{\prime}(3)$ & 13.1261 & 13.126 & & 13.1339 & 13.142 & $c_{3}(1)$ & 0.106 & 0.4041 & $0.391 \pm 0.119$ & $0.391 \pm 0.119$ \\
\hline$b(7)$ & 13.1559 & 13.156 & 13.156 & 13.1504 & 13.156 & $c_{3}(2)$ & 0.126 & 0.0952 & $0.110 \pm 0.017$ & $0.110 \pm 0.017$ \\
\hline$G(1)$ & 13.1625 & & & & 13.06 & $c_{3}(3)$ & 0.007 & 0.0116 & $0.004 \pm 0.001$ & $0.004 \pm 0.001$ \\
\hline$c_{4}^{\prime}(1)$ & 13.1881 & 13.188 & & 13.216 & 13.185 & & & & & \\
\hline$c_{3}(1)$ & 13.2078 & 13.208 & 13.215 & 13.211 & 13.210 & $o_{3}(0)$ & 0.008 & 0.0156 & $0.026 \pm 0.026$ & $0.024 \pm 0.023$ \\
\hline$F(1)$ & 13.212 & & & & 13.210 & $o_{3}(1)$ & 0.201 & 0.4076 & $0.377 \pm 0.162$ & $0.343 \pm 0.148$ \\
\hline$b^{\prime}(4)$ & 13.2225 & 13.222 & & 13.2242 & 13.232 & $o_{3}(2)$ & 0.421 & 0.3818 & $0.245 \pm 0.190$ & $0.224 \pm 0.173$ \\
\hline$b(8)$ & 13.258 & 13.255 & 13.258 & 13.2474 & 13.260 & $o_{3}(3)$ & 0.370 & 0.1950 & $0.352 \pm 0.237$ & $0.321 \pm 0.216$ \\
\hline$b^{\prime}(5)$ & 13.3068 & 13.307 & & 13.3137 & 13.322 & & & & & \\
\hline$o_{3}(1)$ & 13.3445 & 13.345 & 13.346 & 13.346 & 13.345 & $b^{\prime}(0)$ & 0.012 & 0.0000 & $0.108 \pm 0.030$ & $0.009 \pm 0.002$ \\
\hline$b(9)$ & 13.3468 & 13.347 & 13.346 & 13.3429 & 13.345 & $b^{\prime}(1)$ & 0.024 & 0.0262 & $0.142 \pm 0.176$ & $0.011 \pm 0.014$ \\
\hline$D(2)$ & 13.3779 & & & 13.3791 & & $b^{\prime}(2)$ & 0.020 & 0.0033 & $0.016 \pm 0.009$ & $0.001 \pm 0.001$ \\
\hline$b^{\prime}(6)$ & 13.3902 & 13.390 & 13.396 & 13.4022 & 13.410 & $b^{\prime}(3)$ & 0.014 & 0.0067 & $0.050 \pm 0.013$ & $0.004 \pm 0.001$ \\
\hline$G(2)$ & 13.394 & & 13.396 & & & $b^{\prime}(4)$ & 0.072 & 0.1126 & $0.042 \pm 0.033$ & $0.003 \pm 0.003$ \\
\hline$b(10)$ & 13.4374 & 13.438 & 13.437 & 13.4375 & 13.435 & $b^{\prime}(5)$ & 0.010 & 0.0518 & $0.037 \pm 0.009$ & $0.003 \pm 0.001$ \\
\hline$c_{4}^{\prime}(2)$ & 13.4579 & 13.456 & & 13.478 & 13.459 & $b^{\prime}(6)$ & 0.024 & 0.0768 & $0.066 \pm 0.019$ & $0.005 \pm 0.002$ \\
\hline$F(2)$ & 13.468 & & & & 13.430 & $b^{\prime}(7)$ & 0.206 & 0.0107 & $0.032 \pm 0.012$ & $0.003 \pm 0.001$ \\
\hline$c_{3}(2)$ & 13.4764 & 13.476 & 13.477 & 13.476 & 13.475 & $b^{\prime}(8)$ & 0.028 & 0.1507 & $0.244 \pm 0.133$ & $0.020 \pm 0.011$ \\
\hline$b^{\prime}(7)$ & 13.5082 & 13.507 & & 13.4899 & 13.498 & $b^{\prime}(9)$ & 0.270 & 0.3665 & $0.170 \pm 0.040$ & $0.014 \pm 0.003$ \\
\hline$b(11)$ & 13.5291 & 13.530 & & 13.5280 & 13.530 & $b^{\prime}(10)$ & 0.320 & 0.1947 & $0.094 \pm 0.028$ & $0.008 \pm 0.002$ \\
\hline$b^{\prime}(8)$ & 13.5817 & 13.582 & 13.582 & 13.577 & 13.583 & & & & & \\
\hline$o_{3}(2)$ & 13.5839 & 13.583 & 13.584 & 13.585 & 13.585 & $c_{4}^{\prime}(0)$ & 0.932 & 0.8966 & $0.952 \pm 0.030$ & $0.785 \pm 0.025$ \\
\hline
\end{tabular}


TABLE I. (Continued.)

\begin{tabular}{|c|c|c|c|c|c|c|c|c|c|c|}
\hline \multirow{2}{*}{$\begin{array}{l}\text { (a) } \\
\text { Level }\end{array}$} & \multicolumn{5}{|c|}{ Excitation energies $(\mathrm{eV})$} & \multirow{2}{*}{$\begin{array}{c}\text { (b) } \\
\text { Level }\end{array}$} & \multicolumn{4}{|c|}{ Relative excitation probabilities (REPs) } \\
\hline & $\mathrm{P}$ & SLD & $\mathrm{H}$ & LK & $\mathrm{T}$ & & $\mathrm{T}$ & SLD & $\mathrm{P}$ & $\mathrm{P}$ (renorm) \\
\hline$b(12)$ & 13.6174 & 13.619 & & 13.6172 & 13.615 & $c_{4}^{\prime}(1)$ & 0.009 & 0.0530 & $0.041 \pm 0.002$ & $0.034 \pm 0.002$ \\
\hline$D(3)$ & 13.6254 & & & 13.642 & & $c_{4}^{\prime}(2)$ & 0.004 & 0.0075 & $0.002 \pm 0.004$ & $0.002 \pm 0.003$ \\
\hline$G(3)$ & 13.635 & & & & & $c_{4}^{\prime}(3)$ & 0.056 & 0.0429 & $0.005 \pm 0.003$ & $0.004 \pm 0.002$ \\
\hline$b^{\prime}(9)$ & 13.6627 & 13.664 & 13.663 & 13.6627 & 13.668 & & & & & \\
\hline$b(13)$ & 13.7041 & 13.705 & & 13.7041 & 13.700 & $D(0)$ & 0.982 & & $0.985 \pm 0.246$ & \\
\hline$c_{4}^{\prime}(3)$ & 13.7207 & 13.720 & 13.720 & 13.734 & 13.720 & $D(1)$ & 0.018 & & $0.015 \pm 0.004$ & \\
\hline$F(3)$ & 13.729 & & & & & $D(2)$ & 0.000 & & $0.000 \pm 0.000$ & \\
\hline$c_{3}(3)$ & 13.7378 & 13.737 & 13.737 & 13.737 & 13.722 & $D(3)$ & 0.000 & & $0.000 \pm 0.000$ & \\
\hline$b^{\prime}(10)$ & 13.7554 & 13.754 & 13.755 & 13.7478 & 13.751 & $G(0)$ & 0.500 & & $0.603 \pm 0.151$ & \\
\hline$b(14)$ & 13.7884 & 13.790 & & 13.789 & 13.785 & $G(1)$ & 0.500 & & $0.182 \pm 0.045$ & \\
\hline$o_{3}(3)$ & 13.8194 & 13.818 & & 13.820 & 13.820 & $G(2)$ & 0.000 & & $0.207 \pm 0.052$ & \\
\hline & & & & & & $G(3)$ & 0.000 & & $0.008 \pm 0.002$ & \\
\hline & & & & & & $F(0)$ & 0.264 & & $0.585 \pm 0.146$ & \\
\hline & & & & & & $F(1)$ & 0.373 & & $0.141 \pm 0.035$ & \\
\hline & & & & & & $F(2)$ & 0.219 & & $0.180 \pm 0.045$ & \\
\hline & & & & & & $F(3)$ & 0.115 & & $0.094 \pm 0.024$ & \\
\hline
\end{tabular}

this range (see Table I) as independent features. (See below for discussion of the choice of energy-loss range.) Transition energies for the $X^{1} \Sigma_{g}^{+}\left(v^{\prime \prime}=0\right) \rightarrow b{ }^{1} \Pi_{u}, \quad c_{3}{ }^{1} \Pi_{u}, \quad o_{3}{ }^{1} \Pi_{u}$, $b^{\prime}{ }^{1} \Sigma_{u}^{+}, c_{4}^{\prime}{ }^{1} \Sigma_{u}^{+}, D^{3} \Sigma_{u}^{+}, G^{3} \Pi_{u}$, and $F^{3} \Pi_{u}$ features in the energy-loss region of interest were taken from a semiempirical coupled-channels Schrodinger-equation (CSE) investigation by one of us (B.R.L.) [45]. In the case of the $a^{\prime \prime}{ }^{1} \Sigma_{g}^{+}$ features, transition energies were taken from a best fit to the experimental data at small $\theta$.

Regions clear of features were used to obtain the background contribution to the inelastic energy-loss spectra. Background contributions were expressed as polynomials of up to order 2, which were dependent on the incident electron current $I_{0}$ and the target gas density distribution $\rho$ over the appropriate energy-loss region:

$$
B\left(E_{0}, \theta, E_{i}, \rho, I_{0}\right)=\sum_{k=0}^{k<3} B_{k} E_{i}^{k}
$$

The line shape used for fitting each vibrational transition in the energy-loss region was determined empirically by nonlinear least-squares fitting of a multi-Gaussian function to the isolated $a^{\prime \prime}{ }^{1} \Sigma_{g}^{+}\left(v^{\prime}=0\right)$ feature. The line function

$F\left(E-E_{n^{\prime}, v^{\prime}}\right)=\sum_{m=1}^{M} \frac{A_{m}}{\Delta_{m} \pi^{1 / 2}} \exp \left[-\left(\frac{E-E_{n^{\prime}, v^{\prime}}-E_{m}}{\Delta_{m}}\right)^{2}\right]$,

where $M \leqslant 2$ and each Gaussian is located off the line center by an energy loss $E_{m}$, with relative intensity $A_{m}$ and width $\Delta_{m}$, was used for determining the line shape. This was found to be consistent with other well-resolved features. In Eq. (3), $E_{n^{\prime}, v^{\prime}}$ is the energy-loss value for the $v^{\prime}$ th vibrational level of the $n^{\prime}$ th electronic state (see Table I).
Hence, the energy-loss region of interest was fit using

$$
\begin{aligned}
S_{i}\left(E_{0}, \theta, E_{i}\right)= & C \sum_{n^{\prime}, v^{\prime}} \sigma_{n^{\prime}, v^{\prime}}\left(E_{0}, \theta\right) F\left(E_{i}-E_{n^{\prime}, v^{\prime}}\right) \\
& +B\left(E_{0}, \theta, E_{i}, \rho, I_{0}\right)
\end{aligned}
$$

to each multichannel spectrum by means of a nonlinear leastsquares algorithm to all features appertaining to the $n^{\prime}$ th electronic state with its $v^{\prime}$ th vibrational level at the energyloss for the $i$ th channel or bin. The overall fitting (using the singular-value decomposition matrix-inversion technique) yields a relative DCS, $C \sigma_{n^{\prime}, v^{\prime}}$, where $C$ is a constant for each spectrum that must be determined by normalization of the spectrum to an absolute DCS standard (as discussed later). In Eqs. (2) and (4), the variables $C \sigma_{n^{\prime}, v^{\prime}}\left(E_{0}, \theta\right)$ and $B_{k}$ were determined by linear least-squares fitting to the spectra. [Note that the absolute DCSs, $\sigma_{n^{\prime}, v^{\prime}}\left(E_{0}, \theta\right)$, were later determined from the relative $C \sigma_{n^{\prime}, v^{\prime}}\left(E_{0}, \theta\right)$ values upon normalization to a known cross section.] By summing the intensities of all features in the energy-loss spectrum, it was possible to determine the fractional intensity of the feature $\left(n^{\prime}, v^{\prime}\right)$ relative to the whole spectrum. Two further adjustments resulted in improved fits to the spectrum.

a. Energy origin adjustment. The energy origin for the nonlinear least-squares fit of Eq. (4) to the experimental spectra could be varied to further minimize the residual of the fit; i.e., the energy loss at the start of the spectrum, $E_{1}$, was changed in successively reduced increments until a minimum in the reduced chi-squared value $\chi_{v}^{2}$ was reached. Importantly, it was observed that this resultant minimum in $\chi_{\nu}^{2}$ was (uniquely) global for the spectrum, even after multiple independent fittings.

b. Energy step size adjustment. The step size $\delta E$ (i.e., 

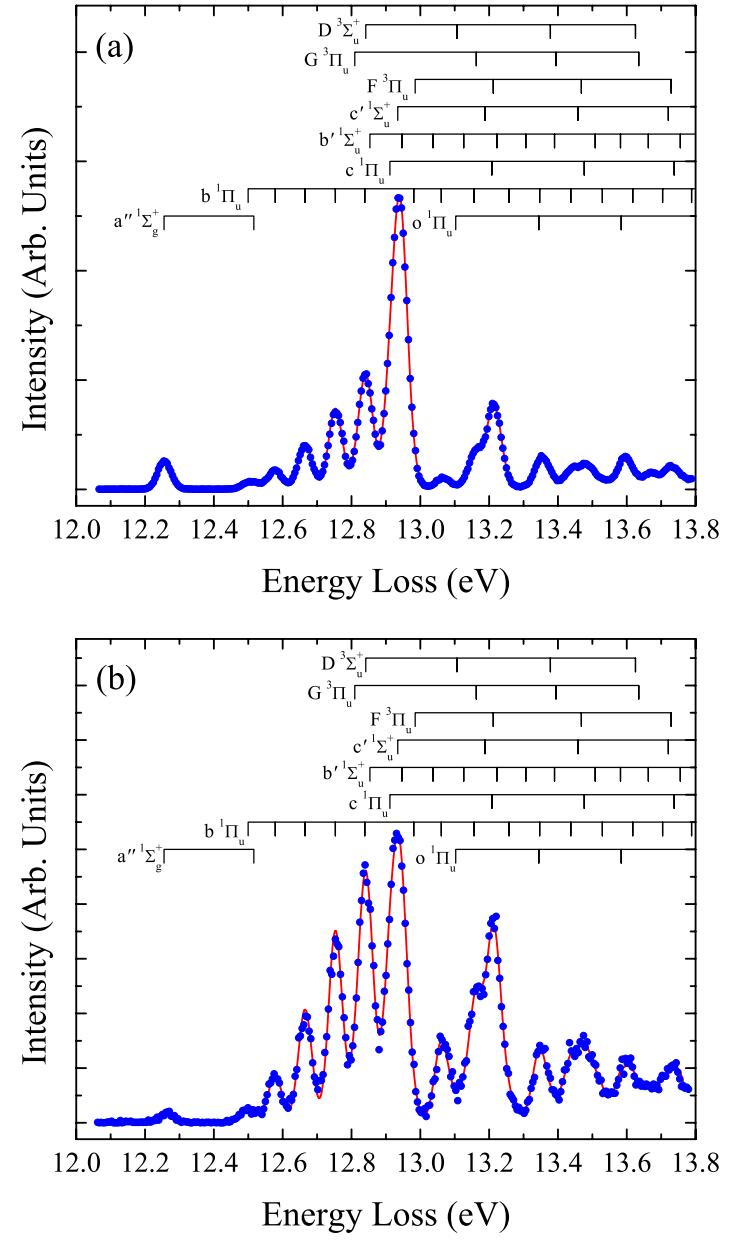

FIG. 2. (Color online) Electron energy-loss spectra of $\mathrm{N}_{2}$ taken at $E_{0}=50 \mathrm{eV}$ and scattering angles of (a) $3^{\circ}$ and (b) $20^{\circ}$. The positions of the various spectral lines are indicated in these figures. Note, although indicated in the figures, the $D^{3} \Sigma_{u}^{+}$state features were too weak to be extracted from the measured spectra (see text). These angles are chosen to highlight the significant relative decrease of the height of the $a^{\prime \prime}{ }^{1} \Sigma_{g}^{+}\left(v^{\prime}=0\right)$ state energy-loss feature (located at $12.255 \mathrm{eV}$ ), in (b), at the DCS minimum, and the significant rise in the $b^{1} \Pi_{u}\left(v^{\prime}=6\right)$ feature, compared with (a). The dots are the present experimental data and the line is a spectral fit using energy-loss data from Table I, present work P.

energy loss per channel) was varied in very small amounts, in association with Sec. II A 2 a above, to minimize $\chi_{\nu}^{2}$. However, the improvements in $\chi_{\nu}^{2}$, which were observed when doing this secondary correction, were significantly smaller than in Sec. II A 2 a, typically by a factor of 10 . This occurs because $\delta E$ did not vary significantly during the course of the experiment; i.e., the linearity of the energy-loss ramp voltage, which determines the energy-loss value, was typically $\pm 2 \mathrm{mV}$ over a $5-\mathrm{V}$ span.

The procedures described in Secs. II A 2 a and II A 2 b above resulted in improved fits, as gauged from $\chi_{\nu}^{2}$ values, which were normally in the range of $1-3$, for fits to spectra with $\sim 800$ data points and $\sim 53$ variables (i.e., with $\approx 747$ degrees of freedom, $\nu$ ). Examples of unfolded spectra are shown in Fig. 2.

An additional complication resulted from the overlap of several energy-loss lines within $\sim 10 \mathrm{meV}$ that could not be separated by the present resolution of our spectrometer ( $\sim 40$ meV FWHM; see Table I and Fig. 2) in conjunction with the present unfolding scheme. Specifically, the following "paired levels" could not be resolved: $b(4)$ and $D(0)$, $b(5)$ and $F(0), o_{3}(0)$ and $D(1), c_{3}(1)$ and $F(1), o_{3}(1)$ and $b(9), b^{\prime}(6)$ and $G(2)$, and $b^{\prime}(8)$ and $o_{3}(2)$. Treatment of these paired levels was carefully considered in the analysis in order to assign the measured spectral intensity to each level within these pairs in as accurate a manner as possible. A detailed description of this treatment is presented in Sec. II B. Increasing the resolution of the spectrometer would have required a reduction of the electron current and consequently a reduction in the scattered electron signal, resulting in an increase in overall statistical uncertainty. The present approach is, therefore, a compromise to obtain as broad a coverage of the line intensities with as much scattering signal as possible (i.e., minimal statistical uncertainty) in a practical amount of time.

Relative excitation probabilities (REPs) were determined from the individual vibronic intensities obtained from the spectral unfolding and are reported in Table I [as the present (P) REP values]. The unfolded intensities were normalized so that the sum of individual vibrational-level intensities for a given electronic state, within our measured energy-loss range, were equal to unity. The same normalization procedure and criteria were independently applied to the derived REPs of Stahel et al. [8] (based on their coupled results) and Trajmar [46] (based on [19,26,30], etc.), with results reported in Table I for direct comparison with the present REP values. Further, the present REPs were normalized a second time so that the sum of individual vibrational-level intensities for a given electronic state were equal to the corresponding sum of REPs of Stahel et al. [8] within our measured energy-loss range. In doing so, we ensure that the present renormalized REPs sum to unity over the entire vibrational manifold of each electronic state when combined with the (coupled) "FC factors" of [8] for vibronic levels outside our energy-loss range. These renormalized REPs are reported in Table I as "P (renorm)" for use in the GOS analysis presented in Sec. III B. Note that the present REPs for the dipole-allowed states are an average taken from unfolding the electron energy-loss spectra obtained at $E_{0}=30,50$, and $100 \mathrm{eV}$, for $\theta \leqslant 3^{\circ}$.

\section{Determining absolute inelastic DCSs}

The method described by Nickel et al. [47] based on elastic electron- $\mathrm{N}_{2}$ DCSs was used to place our total spectral intensities on an absolute DCS scale as follows.

a. Background subtracted inelastic-to-elastric ratios. First, using the movable-source method [42], we simultaneously measured spectra covering the elastic $(-0.25$ to $+0.25 \mathrm{eV}$ ) and inelastic (6 to $14.5 \mathrm{eV}$ ) energy-loss regions. Spectra were measured with the gas source aligned with the electron beam (signal+background; IN) and then moved out of alignment (background; OUT). This was executed for all $E_{0}$ with an angular coverage including $5^{\circ}$ and from $10^{\circ}$ to 
$130^{\circ}$ in $10^{\circ}$ increments. The subtracted results of the OUT spectra from the IN spectra were used to determine the ratios of relative inelastic DCSs of the summed $A+B+W+B^{\prime}+a^{\prime}$ $+a+w+C$ states (region 1; $E$ from 6 to $11.5 \mathrm{eV}$ ) and $a^{\prime \prime}+b$ $+c_{3}+o_{3}+b^{\prime}+c_{4}^{\prime}+D+G+F$ states (region 2; $E$ from 12 to $13.82 \mathrm{eV}$ ) to the relative elastic DCSs. Interpolation $(B$-spline) between measured ratios was performed for each $E_{0}$ over the measured angular range. Not only did this procedure accurately account for background signals (particularly for elastic spectra) using the movable-source method, but also eliminated possible geometrical scattering issues (i.e., angular-dependent scattering volume) via the simultaneously measured inelastic-to-elastic ratios. It should be noted that these spectra were determined using a coarser $\delta E$ compared with the spectra in Sec. II A 2; i.e., fewer channels and scans were used. These spectra were used to generate ratios involving summed regions of the inelastic spectra and were therefore not subject to the unfolding routine described in Sec. II A 2 a. The experimental efforts of Secs. II A 2 and II A 3 a were performed consecutively for each $E_{0}$ to minimize possible system variations, though we found the experiment to be very stable over the course of the work (as previously discussed). Of note, the summed statistical uncertainty of Sec. II A 3 a was always better than $3 \%$ on average after subtraction of the background. We also note that in region 2 , the $E^{3} \Sigma_{g}^{+}\left(v^{\prime}>1\right)$ levels were within the measured energy-loss range. However, the $E(1)$ level, at $12.139 \mathrm{eV}$, did not overlap with the $a^{\prime \prime}(0)$ level (see Table I) $[48,49]$. Further, the FC factors for the $E^{3} \Sigma_{g}^{+}\left(v^{\prime}=0,1\right)$ levels are approximately 0.9278 and $0.0625[48,49]$, respectively, leaving only a summed FC factor of about 0.0097 for all $v^{\prime}>1$, which overlap with the present energy-loss spectra. For the incident electron energies used in the present investigation, any signal attributable to the $E^{3} \Sigma_{g}^{+}$state within our measured energy-loss range was negligible.

$b$. Transmission adjusted inelastic-to-elastic ratios. The summed DCS data, obtained in Sec. II A 3 a, were renormalized using the inelastic-to-elastic DCS ratios of LeClair and Trajmar [43] at $\theta=90^{\circ}$, which have an uncertainty of $<10 \%$. We note that the minimization of transmission effects, discussed in Sec. II A 1 above, improved the quality of the data by requiring relatively small transmission corrections via [43]. We further compared the inelastic-to-elastic ratios for region 1 with our previous measurements [1], which provided consistent results. The normalized inelastic-to-elastic ratios are listed in Table II. Inelastic-to-elastic ratios were then obtained for each unfolded inelastic feature by comparing the individual $\left(n^{\prime}, v^{\prime}\right)$ relative intensities of Sec. II A 2 with the summed intensities of Sec. II A $3 \mathrm{~b}$. This procedure minimizes and eliminates transmission issues and results in improved relative inelastic DCSs over extended energy-loss ranges.

c. Absolute normalization. Absolute inelastic DCSs were then obtained by multiplying the inelastic-to-elastic ratios (Sec. II A 3 b) to an average of selected experimental DCSs for elastic electron scattering from $\mathrm{N}_{2}$ of Srivastava et al. [50] (corrected in [19]), Shyn and Carignan [51] (corrected in [19]), Nickel et al. [52], and Gote and Ehrhardt [53]. These data are tabulated in Trajmar et al. [19] and Brunger and Buckman [54]. In our selection, we used those values of elastic scattering DCSs which agreed within their combined quoted uncertainties. Consequently, absolute inelastic DCSs for each unfolded feature were obtained with the effective absolute inelastic DCSs for each electronic state discussed below (Sec. II B).

Overall, the experimental uncertainty assigned to each quantity is the square root of the sum of the squares of the contributing uncertainty components. For the DCS values associated with the sum of the nine state excitations at $90^{\circ}$, we considered the statistical and fitting uncertainties in the individual scattering intensities (typically 2\%-25\%), the inelastic-to-elastic ratio uncertainty of the TOF results of LeClair and Trajmar $[43](\approx 10 \%)$, the uncertainties in the available elastic-scattering DCSs $(\approx 14 \%)$, the uncertainty propagated by the present inelastic-to-elastic ratio measurements (typically 5\%), and an additional uncertainty of $10 \%$ for the analyzer response function. Finally, we also note here that no smoothing is applied to the final shape of our DCS data. Our DCSs and associated uncertainties are listed in Table III. These DCSs are compared with existing measurements and theoretical values in Figs. 3-9.

\section{B. Additional details}

Unresolved energy-loss features led to (effective) pairings between the levels with italicized excitation energies in Table I. Specifically, the present direct unfolding of the energy-loss spectra could not resolve all levels and therefore yielded combined intensities for the following seven level pairs: $b(4)$ and $D(0), b(5)$ and $F(0), o_{3}(0)$ and $D(1), c_{3}(1)$ and $F(1)$, $o_{3}(1)$ and $b(9), b^{\prime}(6)$ and $G(2)$, and $b^{\prime}(8)$ and $o_{3}(2)$. The intensities associated with each pair were distributed among the paired levels as follows.

The intensities associated with the $o_{3}(1)$ and $b(9)$ and $o_{3}(2)$ and $b^{\prime}(8)$ pairings were applied to the $o_{3}{ }^{1} \Pi_{u}$ state DCS due to the relatively large (coupled) FC factors (i.e., intensities) given by Stahel et al. for the $o_{3}(1)$ and $o_{3}(2)$ levels when compared to those of the $b(9)$ and $b^{\prime}(8)$ levels [8]. As discussed previously, the FC principle is strictly invalid for these transitions. Therefore, partitioning intensities based on FC factors is not feasible in this instance. However, using FC factors, in combination with the number of vibrational levels observed within each manifold, to guide the assignment of intensity to one level or the other represents a "best effort" attempt to address these unresolved levels.

The only dipole-forbidden transitions that could be resolved completely in the present work were excitations to the $G(0), G(1), D(2), D(3), G(3)$, and $F(3)$ levels while the remaining triplet levels formed unresolved pairs with levels of the $b^{1} \Pi_{u}, c_{3}{ }^{1} \Pi_{u}, o_{3}{ }^{1} \Pi_{u}$, and $b^{\prime}{ }^{1} \Sigma_{u}^{+}$states. The FC factors for levels of these dipole-forbidden states (see Table I) were computed from RKR calculations using a code from Gilmore et al. [55] and the molecular constants for these levels listed in Lofthus and Krupenie [16]. Using the measured intensities of the resolved $G(0), G(1), D(2), D(3)$, $G(3)$, and $F(3)$ levels, along with the computed RKR FC factors, the intensities of unresolved $D^{3} \Sigma_{u}^{+}, G^{3} \Pi_{u}$, and $F^{3} \Pi_{u}\left(v^{\prime}\right)$ levels were estimated and subtracted from the ap- 
TABLE II. Inelastic-to-elastic ratios $R$ for electron scattering from $\mathrm{N}_{2}$, determined using the movable-source method [42]. The elastic DCSs used were the same as those used in our earlier work [1] and can be computed from this table. The inelastic ratio spans the energy-loss window of 12-13.82 eV, covering the range of levels given in Table I. Total inelastic DCSs are given in units of $10^{-18} \mathrm{~cm}^{2} \mathrm{sr}^{-1}$.

\begin{tabular}{|c|c|c|c|c|c|c|c|c|c|c|}
\hline \multirow{2}{*}{$\begin{array}{l}\text { Angle } \\
\text { (degrees) }\end{array}$} & \multicolumn{2}{|c|}{$17.5 \mathrm{eV}$} & \multicolumn{2}{|c|}{$20 \mathrm{eV}$} & \multicolumn{2}{|c|}{$30 \mathrm{eV}$} & \multicolumn{2}{|c|}{$50 \mathrm{eV}$} & \multicolumn{2}{|c|}{$100 \mathrm{eV}$} \\
\hline & $R$ & Total DCS & $R$ & Total DCS & $R$ & Total DCS & $R$ & Total DCS & $R$ & Total DCS \\
\hline 2 & & & & & & & & & 0.749 & 1000 \\
\hline 3 & & & & & & & 0.296 & 325 & 0.594 & 747 \\
\hline 5 & & & 0.0298 & 20.1 & 0.0772 & 77.8 & 0.228 & 235 & 0.344 & 378 \\
\hline 8 & & & & & & & 0.161 & 153 & 0.181 & 159 \\
\hline 10 & 0.00709 & 3.83 & 0.0232 & 14.1 & 0.0619 & 52.1 & 0.133 & 114 & 0.111 & 82.2 \\
\hline 12 & & & & & & & 0.106 & 80 & 0.068 & 42.3 \\
\hline 15 & 0.00734 & 3.47 & 0.0199 & 10.7 & 0.0501 & 33.8 & 0.0738 & 44.8 & 0.0432 & 20.4 \\
\hline 18 & & & & & & & & & 0.0328 & 11.4 \\
\hline 20 & 0.00758 & 3.06 & 0.0192 & 8.72 & 0.04 & 20.5 & 0.0477 & 19.7 & 0.0306 & 8.59 \\
\hline 25 & 0.00734 & 2.44 & 0.0168 & 6.09 & 0.0316 & 11.6 & 0.0398 & 11.3 & 0.0355 & 5.52 \\
\hline 30 & 0.00722 & 1.93 & 0.0152 & 4.21 & 0.0294 & 7.48 & 0.0402 & 6.76 & 0.0477 & 3.88 \\
\hline 35 & 0.0073 & 1.56 & 0.0147 & 3.08 & 0.0293 & 5.23 & 0.0488 & 5.45 & 0.0571 & 2.52 \\
\hline 40 & 0.00734 & 1.26 & 0.0156 & 2.5 & 0.0315 & 3.98 & 0.0575 & 3.87 & 0.0574 & 1.59 \\
\hline 45 & 0.00839 & 1.15 & 0.0161 & 2 & 0.0394 & 3.48 & 0.0678 & 3.11 & 0.0546 & 1.05 \\
\hline 50 & 0.00995 & 1.1 & 0.0182 & 1.77 & 0.0502 & 3.12 & 0.0769 & 2.5 & 0.0479 & 0.687 \\
\hline 55 & & & & & 0.0583 & 2.64 & & & 0.0465 & 0.543 \\
\hline 60 & 0.0141 & 1.01 & 0.0269 & 1.57 & 0.0683 & 2.33 & 0.0717 & 1.27 & 0.0429 & 0.433 \\
\hline 65 & & & & & & & & & 0.039 & 0.346 \\
\hline 70 & 0.0228 & 1.02 & 0.0384 & 1.47 & 0.105 & 2.04 & 0.0907 & 1.01 & & \\
\hline 80 & 0.0351 & 1.04 & 0.0614 & 1.46 & 0.133 & 1.84 & 0.0947 & 0.738 & & \\
\hline 90 & 0.0345 & 0.812 & 0.0649 & 1.32 & 0.135 & 1.58 & 0.0981 & 0.598 & & \\
\hline 100 & 0.024 & 0.673 & 0.0493 & 1.31 & 0.121 & 1.61 & 0.0756 & 0.559 & & \\
\hline 110 & 0.0174 & 0.666 & 0.0338 & 1.27 & 0.079 & 1.5 & 0.0557 & 0.618 & & \\
\hline 120 & 0.0131 & 0.663 & 0.0261 & 1.3 & 0.0603 & 1.69 & 0.0448 & 0.865 & & \\
\hline 130 & 0.0115 & 0.736 & 0.022 & 1.33 & 0.0425 & 1.78 & 0.0379 & 1.18 & & \\
\hline $\begin{array}{l}\text { Fractional } \\
\text { error }\end{array}$ & 0.087 & 0.165 & 0.080 & 0.161 & 0.085 & 0.164 & 0.085 & 0.164 & 0.089 & 0.166 \\
\hline
\end{tabular}

propriate levels of the $b^{1} \Pi_{u}, c_{3}{ }^{1} \Pi_{u}, o_{3}{ }^{1} \Pi_{u}$, and $b^{\prime}{ }^{1} \Sigma_{u}^{+}$ states. Further, the resolved components of the forbidden transitions-i.e., $G(0), G(1), D(2), D(3), G(3)$, and $F(3)$, were used to extricate full-state DCSs for the $G^{3} \Pi_{u}$ and $F^{3} \Pi_{u}$ states, which represents a best effort attempt at unfolding the state specific DCSs from the complicated energy-loss data. Because of the suspected strong mixing among levels of the $G^{3} \Pi_{u}$ and $F^{3} \Pi_{u}$ states [9], and associated interference effects, this likely leads to some inaccuracy in these procedures, but at present, there is no reliable alternative available.

The procedure described in the previous paragraph was further complicated in the case of the $D^{3} \Sigma_{u}^{+}$state due to the weakness of the $D^{3} \Sigma_{u}^{+}$state transitions. In fact, the unfolding procedure did not attribute any measurable intensity to the otherwise resolvable $D(2)$ and $D(3)$ features. This result was used to set an upper limit on the intensity attributable to the $D(0)$ and $D(1)$ levels based on the calculated RKR FC factors. On this basis, small contributions of the $D(0)$ and $D(1)$ levels were estimated and subtracted from the $b(4)$ and $o_{3}(0)$ levels, respectively.
To improve the overall picture regarding the $c_{4}^{\prime}(0)$ and $b^{\prime}(1)$ levels, which are nearing the resolution limit of our measurement and unfolding procedures, additional "high"resolution energy-loss data $(<35 \mathrm{meV}$ FWHM) at small angles $\left(<30^{\circ}\right)$ were analyzed. At small angles, there were sufficient statistics and resolution to accurately unfold the contributions of the optically allowed $c_{4}^{\prime}(0)$ and $b^{\prime}(1)$ transitions. These more accurately determined intensity distributions were used to correct previously determined DCSs of the $b^{\prime}{ }^{1} \Sigma_{u}^{+}$and $c_{4}^{\prime}{ }^{1} \Sigma_{u}^{+}$states. The remaining states were adjusted uniformly such that the total summed DCS remained unchanged.

In summary, the present direct unfolding of the energyloss spectra yielded DCSs for the excitation of the partial $b^{1} \Pi_{u}$ state $[b=b(0)+\cdots+b(14)]$, essentially full $c_{3}{ }^{1} \Pi_{u}$ state $\left[c_{3}=c_{3}(0)+\cdots+c_{3}(3)\right]$, partial $o_{3}{ }^{1} \Pi_{u}$ state $\left[o_{3}=o_{3}(0)\right.$ $\left.+\cdots+o_{3}(3)\right]$, partial $b^{\prime}{ }^{1} \Sigma_{u}^{+}$state $\left[b^{\prime}=b^{\prime}(0)+\cdots+b^{\prime}(10)\right]$, partial $c_{4}^{\prime}{ }^{1} \Sigma_{u}^{+}$state $\left[c_{4}^{\prime}=c_{4}^{\prime}(0)+\cdots+c_{4}^{\prime}(3)\right]$, essentially full $G^{3} \Pi_{u}$ state $[G=G(0)+\cdots+G(3)]$, and essentially full $F^{3} \Pi_{u}$ state $[F=F(0)+\cdots+F(3)]$. 
TABLE III. Differential cross sections for the electron-impact excitation of the $X^{1} \Sigma_{g}^{+}\left(v^{\prime \prime}=0\right) \rightarrow a^{\prime \prime}{ }^{1} \Sigma_{g}^{+}, b^{1} \Pi_{u}, c_{3}{ }^{1} \Pi_{u}, o_{3}{ }^{1} \Pi_{u}, b^{\prime}{ }^{1} \Sigma_{u}^{+}$, $c_{4}^{\prime}{ }^{1} \Sigma_{u}^{+}, G^{3} \Pi_{u}$, and $F^{3} \Pi_{u}$ transitions in $\mathrm{N}_{2}$. (a) $E_{0}=17.5 \mathrm{eV}$, (b) $E_{0}=20 \mathrm{eV}$, (c) $E_{0}=30 \mathrm{eV}$, (d) $E_{0}=50 \mathrm{eV}$, and (e) $E_{0}=100 \mathrm{eV}$. Error bars include the uncertainties in the elastic electron-scattering DCS (14\%), elastic-to-inelastic transmission errors (5\%-10\%), and the spectral unfolding process $(10 \%-25 \%)$. The average uncertainty is given at the bottom of each column. See text for details regarding vibrational coverage in the last paragraph of Sec. II B.

\begin{tabular}{|c|c|c|c|c|c|c|c|c|c|c|c|c|c|c|c|c|c|}
\hline \multirow{2}{*}{$\begin{array}{l}\text { Angle } \\
\text { (degrees) }\end{array}$} & \multicolumn{2}{|c|}{ Total } & \multicolumn{2}{|c|}{$a^{\prime \prime}{ }^{1} \Sigma_{g}^{+}$} & \multicolumn{2}{|c|}{$b^{1} \Pi_{u}$} & \multicolumn{2}{|c|}{$c_{3}{ }^{1} \Pi_{u}$} & \multicolumn{2}{|c|}{$o_{3}{ }^{1} \Pi_{u}$} & \multicolumn{2}{|c|}{$b^{\prime}{ }^{1} \Sigma_{u}^{+}$} & \multicolumn{2}{|c|}{$c_{4}^{\prime}{ }^{1} \Sigma_{u}^{+}$} & \multicolumn{2}{|c|}{$G^{3} \Pi_{u}$} & $F^{3} \Pi_{u}$ \\
\hline & DCS & rror & DCS & Error & DCS & Error & DCS & Error & DCS & Error & DCS & Erro & D & Error & DCS & Error & DCS Error \\
\hline
\end{tabular}

18

30

35

40

45

50

55

60

65

70

75

80

85

90

95

100

105

110

115

120

125

130

Fractional error $\begin{array}{lllll}3.96 & 0.65 & 0.579 & 0.104 & 1.32\end{array}$

$\begin{array}{lllll}3.91 & 0.64 & 0.873 & 0.157 & 1.20\end{array}$

$\begin{array}{lllll}3.83 & 0.63 & 0.850 & 0.153 & 1.13\end{array}$

$\begin{array}{lllll}3.70 & 0.61 & 0.735 & 0.132 & 1.19\end{array}$

$\begin{array}{lllll}3.47 & 0.57 & 0.562 & 0.101 & 1.23\end{array}$

$\begin{array}{lllll}3.25 & 0.54 & 0.474 & 0.085 & 1.16\end{array}$

$\begin{array}{lllll}3.06 & 0.50 & 0.400 & 0.072 & 1.14\end{array}$

$\begin{array}{llllll}2.44 & 0.40 & 0.259 & 0.046 & 0.858\end{array}$

$\begin{array}{llll}2.24 & 0.37 & 0.222 & 0.040\end{array}$

$\begin{array}{llllll}1.93 & 0.32 & 0.193 & 0.035 & 0.6\end{array}$

$1.56 \quad 0.26$

$\begin{array}{lllll}1.37 & 0.23 & 0.167 & 0.030 & 0.4\end{array}$

$\begin{array}{lllll}1.26 & 0.21 & 0.192 & 0.035 & 0.4\end{array}$

$1.10 \quad 0.18$

$1.03 \quad 0.17$

$1.01 \quad 0.17$

$1.03 \quad 0.17$

$1.02 \quad 0.17$

$1.04 \quad 0.17$

$0.901 \quad 0.149$

$0.812 \quad 0.134$

$0.724 \quad 0.119$

$\begin{array}{lll}0.673 & 0.111 & 0.0896\end{array}$

$\begin{array}{lll}0.679 & 0.112 & 0.109\end{array}$

$\begin{array}{lll}0.666 & 0.110 & 0.116\end{array}$

$\begin{array}{lll}0.664 & 0.110 & 0.118\end{array}$

$\begin{array}{lll}0.663 & 0.109 & 0.137\end{array}$

$\begin{array}{lll}0.674 & 0.111 & 0.129\end{array}$

0.736
0.12

0.152 (1)

$\begin{array}{llll}0.26 & 0.672 & 0.138 & 0.276\end{array}$

0.24

0.22

0.23

0.24

0.23

0.22

0.181

$0.545 \quad 0.112 \quad 0.223$

$\begin{array}{lll}0.534 & 0.109 & 0.192 \\ 0.407 & 0.083 & 0.205\end{array}$

$0.374 \quad 0.076 \quad 0.168$

$\begin{array}{lll}0.260 & 0.053 & 0.161\end{array}$

0.097

$0.475 \quad 0.093$

$\begin{array}{lll}0.184 & 0.038 & 0.141\end{array}$

$\begin{array}{ll}0.036 & 0.104\end{array}$

0.3360 .066

0.113

$0.258 \quad 0.050$

.113

$0.308 \quad 0.060$

$0.027 \quad 0.205$

0.20 $\begin{array}{lllll}1.15 & 0.19 & 0.176 & 0.032 & 0.33\end{array}$

$1.06 \quad 0.17$

\section{(a) $17.5 \mathrm{eV}$ \\ $\begin{array}{llll}0.29 & 0.742 & 0.152 & 0.301\end{array}$}

$\begin{array}{lll}0.522 & 0.107 & 0.219\end{array}$

$\begin{array}{lll}0.627 & 0.128 & 0.206\end{array}$

$\begin{array}{lll}0.563 & 0.115 & 0.205\end{array}$

$\begin{array}{lll}0.514 & 0.105 & 0.216\end{array}$

$0.067 \quad 0.578$

0.062

$0.049 \quad 0.291$

$\begin{array}{lllllll}0.135 & 0.632 & 0.150 & 0.0609 & 0.0160 & 0.0471 & 0.0131\end{array}$

0.046

$0.046 \quad 0.334$

$0.050 \quad 0.301$

.048

$0.043 \quad 0.25$

$0.046 \quad 0.27$

$0.038 \quad 0.202$

0.036

$0.032 \quad 0.192$

$0.023 \quad 0.17$

$\begin{array}{llll}0.028 & 0.105 & 0.023 & 0.162\end{array}$

$\begin{array}{lllll}0.132 & 0.027 & 0.104 & 0.023 & 0.143\end{array}$

$\begin{array}{llll}0.023 & 0.0923 & 0.0207 & 0.147\end{array}$

$\begin{array}{llll}0.023 & 0.0866 & 0.0194 & 0.134\end{array}$

$\begin{array}{llllll}0.021 & 0.0741 & 0.0166 & 0.134\end{array}$

$\begin{array}{llllll}0.139 & 0.029 & 0.0795 & 0.0178 & 0.116\end{array}$

$0.095 \quad 0.537$

$0.127 \quad 0.06$

0939

$\begin{array}{lll}0.0247 & 0.0665 & 0.0185\end{array}$

$\begin{array}{llllllll}0.077 & 0.447 & 0.106 & 0.149 & 0.039 & 0.0833 & 0.0232\end{array}$

$\begin{array}{lllllll}0.078 & 0.419 & 0.099 & 0.163 & 0.043 & 0.0930 & 0.0259\end{array}$

$\begin{array}{llllllll}0.070 & 0.340 & 0.080 & 0.179 & 0.047 & 0.0930 & 0.0259\end{array}$

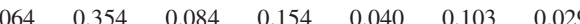

$\begin{array}{lllllll}059 & 0.269 & 0.064 & 0.170 & 0.045 & 0.102 & 0.028\end{array}$

$\begin{array}{lllllll}0.064 & 0.180 & 0.043 & 0.171 & 0.045 & 0.0866 & 0.0241\end{array}$

$\begin{array}{llllllll}0.047 & 0.103 & 0.024 & 0.162 & 0.043 & 0.0865 & 0.0241\end{array}$

$\begin{array}{lllllllll}1 & 0.054 & 0.123 & 0.029 & 0.197 & 0.052 & 0.0944 & 0.0263\end{array}$

$\begin{array}{llllllll}0.045 & 0.0800 & 0.0190 & 0.200 & 0.053 & 0.0790 & 0.0220\end{array}$

$\begin{array}{llllllll}0.040 & 0.0509 & 0.0121 & 0.155 & 0.041 & 0.0673 & 0.0187\end{array}$

$\begin{array}{llllllll}0.038 & 0.0415 & 0.0098 & 0.148 & 0.039 & 0.0638 & 0.0178\end{array}$

$\begin{array}{llllllll}0.034 & 0.0460 & 0.0109 & 0.175 & 0.046 & 0.0794 & 0.0221\end{array}$

$\begin{array}{llllllll}0.031 & 0.0498 & 0.0118 & 0.170 & 0.045 & 0.0678 & 0.0189\end{array}$

$\begin{array}{llllllll}0.031 & 0.0455 & 0.0108 & 0.151 & 0.040 & 0.0747 & 0.0208\end{array}$

$\begin{array}{llllllll}0.027 & 0.0430 & 0.0102 & 0.151 & 0.040 & 0.0729 & 0.0203\end{array}$

$\begin{array}{llllllllllllll}0.317 & 0.062 & 0.141 & 0.029 & 0.0813 & 0.0182 & 0.106 & 0.025 & 0.0638 & 0.0151 & 0.138 & 0.036 & 0.0582 & 0.0162\end{array}$

$\begin{array}{lllllllllllllll}0.359 & 0.070 & 0.140 & 0.029 & 0.0980 & 0.0219 & 0.107 & 0.025 & 0.0543 & 0.0129 & 0.128 & 0.034 & 0.0622 & 0.0173\end{array}$

$\begin{array}{lllllllllllllll}0.377 & 0.074 & 0.126 & 0.026 & 0.100 & 0.022 & 0.120 & 0.028 & 0.0554 & 0.0131 & 0.105 & 0.028 & 0.0476 & 0.0132\end{array}$

$\begin{array}{lllllllllllllll}0.335 & 0.066 & 0.0995 & 0.0204 & 0.0963 & 0.0216 & 0.0938 & 0.0218 & 0.0541 & 0.0128 & 0.0827 & 0.0218 & 0.0334 & 0.0093\end{array}$

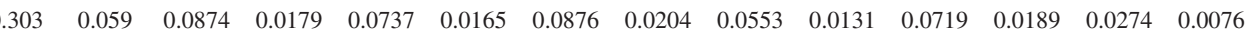

$\begin{array}{lllllllllllllll}0.270 & 0.053 & 0.0779 & 0.0159 & 0.0719 & 0.0161 & 0.0768 & 0.0179 & 0.0417 & 0.0099 & 0.0616 & 0.0162 & 0.0305 & 0.0085\end{array}$

$\begin{array}{llllllllllllllll}0.251 & 0.049 & 0.0808 & 0.0165 & 0.0594 & 0.0133 & 0.0666 & 0.0155 & 0.0479 & 0.0114 & 0.0491 & 0.0129 & 0.0283 & 0.0079\end{array}$

$\begin{array}{llllllllllllllllll}0.020 & 0.243 & 0.048 & 0.0645 & 0.0132 & 0.0640 & 0.0143 & 0.0693 & 0.0161 & 0.0450 & 0.0107 & 0.0531 & 0.0140 & 0.0308 & 0.0086\end{array}$

$\begin{array}{llllllllllllllll}.021 & 0.227 & 0.045 & 0.0625 & 0.0128 & 0.0635 & 0.0142 & 0.0904 & 0.0210 & 0.0320 & 0.0076 & 0.0411 & 0.0108 & 0.0294 & 0.0082\end{array}$

$\begin{array}{llllllllllllllll}0.025 & 0.213 & 0.042 & 0.0556 & 0.0114 & 0.0753 & 0.0169 & 0.0828 & 0.0193 & 0.0283 & 0.0067 & 0.0432 & 0.0114 & 0.0281 & 0.0078\end{array}$

$\begin{array}{lllllll}0.040 & 0.0724 & 0.0148 & 0.0782 & 0.0175 & 0.0960\end{array}$

0.224

0.0223

0.233

0.0104

$0.0163 \quad 0.0262 \quad 0.0073$

(b) $20 \mathrm{eV}$

23.0

1.25

3.10

$0.67+\frac{10}{2}+28$

$\begin{array}{lllll}20.1 & 3.2 & 1.85 & 0.33 & 5.67\end{array}$

$\begin{array}{llllll}16.6 & 2.7 & 2.19 & 0.39 & 5.06 & 0.95\end{array}$

$\begin{array}{lllll}14.1 & 2.3 & 1.74 & 0.31 & 4.79\end{array}$

$\begin{array}{lllll}13.8 & 2.2 & 1.42 & 0.25 & 4.72\end{array}$

$\begin{array}{lllll}10.7 & 1.7 & 0.886 & 0.156 & 4.08\end{array}$

$\begin{array}{lllll}9.51 & 1.53 & 0.648 & 0.114 & 3.77\end{array}$

$\begin{array}{lllll}8.72 & 1.41 & 0.475 & 0.084 & 3.84\end{array}$

$\begin{array}{lllll}6.09 & 0.98 & 0.222 & 0.039 & 2.75\end{array}$

$\begin{array}{lllll}5.04 & 0.81 & 0.300 & 0.053 & 2.03\end{array}$

$\begin{array}{llllll}4.21 & 0.68 & 0.260 & 0.046 & 1.84 & 0.35\end{array}$

$\begin{array}{llllll}3.08 & 0.50 & 0.287 & 0.051 & 1.26 & 0.2\end{array}$

$\begin{array}{llllll}2.78 & 0.45 & 0.310 & 0.055 & 1.16 & 0.22\end{array}$

$\begin{array}{llllll}2.50 & 0.40 & 0.317 & 0.056 & 1.01 & 0.1\end{array}$

$\begin{array}{llllll}2.00 & 0.32 & 0.289 & 0.051 & 0.773 & 0.146\end{array}$
4.68

$0.91 \quad 2.53$

0.53

$\begin{array}{llll}3.86 & 0.75 & 2.10 & 0.44\end{array}$

$\begin{array}{llll}2.85 & 0.56 & 1.49 & 0.31\end{array}$

2.43

2.51

2.13

$0.47 \quad 1.09$

$0.49 \quad 1.03$

$0.53 \quad 3.23$

0.97

0.570

(153

$\begin{array}{lll}1.65 & 0.32 & 0.729\end{array}$

0.73

1.60

1.10

$0.31 \quad 0.584$

$\begin{array}{lll}0.594 & 0.116 & 0.335\end{array}$

$\begin{array}{lll}0.365 & 0.071 & 0.259\end{array}$

$\begin{array}{lll}0.271 & 0.053 & 0.194\end{array}$

$\begin{array}{lll}0.265 & 0.052 & 0.155\end{array}$

$\begin{array}{lll}0.243 & 0.047 \quad 0.131\end{array}$
$0.969-0.189-0.439$
$0.31 \quad 1.97$

$0.21 \quad 1.27$

$0.28 \quad 2.09$

0.45

0.48

0.58

$\begin{array}{ll}0.311 & 0.085\end{array}$

$\begin{array}{llll}0.417 & 0.112 & 0.235 & 0.064\end{array}$

$\begin{array}{llll}0.548 & 0.147 & 0.187 & 0.051\end{array}$

$\begin{array}{lllll}0.396 & 0.107 & 0.184 & 0.050\end{array}$

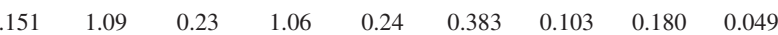

$\begin{array}{lllllllll}0.121 & 0.864 & 0.187 & 0.810 & 0.184 & 0.379 & 0.102 & 0.173 & 0.047\end{array}$

$\begin{array}{lllllllll}0.101 & 0.646 & 0.140 & 0.442 & 0.100 & 0.319 & 0.086 & 0.124 & 0.034\end{array}$

$\begin{array}{llllllllll}0.091 & 0.563 & 0.122 & 0.352 & 0.080 & 0.255 & 0.069 & 0.125 & 0.034\end{array}$

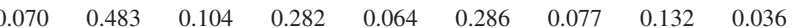

$\begin{array}{lllllllll}0.032 & 0.205 & 0.044 & 0.0994 & 0.0226 & 0.304 & 0.082 & 0.149 & 0.041\end{array}$

$\begin{array}{llllllllll}0.027 & 0.161 & 0.035 & 0.0806 & 0.0183 & 0.217 & 0.058 & 0.104 & 0.028\end{array}$ $\begin{array}{lllllllll}0.054 & 0.390 & 0.084 & 0.133 & 0.030 & 0.270 & 0.072 & 0.123 & 0.034\end{array}$

$\begin{array}{lllllllll}0.040 & 0.279 & 0.060 & 0.107 & 0.024 & 0.309 & 0.083 & 0.149 & 0.041\end{array}$ 
TABLE III. (Continued.)

\begin{tabular}{|c|c|c|c|c|c|c|c|c|c|c|c|c|c|c|c|c|c|c|}
\hline \multirow{2}{*}{$\begin{array}{l}\text { Angle } \\
\text { (degrees) }\end{array}$} & \multicolumn{2}{|c|}{ Total } & \multicolumn{2}{|c|}{$a^{\prime \prime}{ }^{1} \Sigma_{g}^{+}$} & \multicolumn{2}{|c|}{$b{ }^{1} \Pi_{u}$} & \multicolumn{2}{|c|}{$c_{3}{ }^{1} \Pi_{u}$} & \multicolumn{2}{|c|}{$o_{3}{ }^{1} \Pi_{u}$} & \multicolumn{2}{|c|}{$b^{\prime}{ }^{1} \Sigma_{u}^{+}$} & \multicolumn{2}{|c|}{$c_{4}^{\prime}{ }^{1} \Sigma_{u}^{+}$} & \multicolumn{2}{|c|}{$G^{3} \Pi_{u}$} & \multicolumn{2}{|c|}{$F^{3} \Pi_{u}$} \\
\hline & DCS & Error & $\mathrm{CS}$ & Error & DCS & Error & DCS & Error & DCS & Error & DCS & Error & DCS & Error & DCS & Error & DCS & Error \\
\hline 0 & 1.77 & 0.29 & 0.234 & 0.041 & 0.699 & 0.132 & 0.238 & 0.046 & 0.145 & 0.030 & 0.127 & 0.027 & 0.0827 & 0.0188 & 0.150 & 0.040 & 0.0938 & 0.0256 \\
\hline כ3 & 1.65 & 0.27 & 0.224 & 0.039 & 0.612 & 0.116 & 0.214 & 0.042 & 0.130 & 0.027 & 0.128 & 0.028 & 0.0961 & 0.0218 & 0.145 & 0.039 & 0.0963 & 0.0263 \\
\hline 50 & 1.57 & 0.25 & 0.192 & 0.034 & 3 & 0.114 & 45 & 0.048 & 0.127 & 0.026 & 0.102 & 0.022 & 0.0993 & 0.0226 & 0.119 & 0.032 & 0.0810 & 0.0221 \\
\hline 5 & 1.52 & 0.25 & 0.185 & 0.033 & 0.570 & 0.108 & 0.248 & 0.048 & 0.115 & 0.024 & 0.0905 & 0.0196 & 116 & 0.026 & 0.120 & 0.032 & 0.0744 & 0.0203 \\
\hline 70 & 1.47 & 0.24 & 0.160 & 0.028 & 0.587 & 0.111 & 0.222 & 0.043 & 0.110 & 0.023 & 0.113 & 0.025 & 0.106 & 0.024 & 0.103 & 0.028 & 0.0710 & 0.0194 \\
\hline 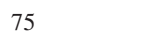 & 1.46 & 0.24 & 0.143 & 0.025 & 0.637 & 0.120 & 0.218 & 0.042 & 0.124 & 0.026 & 0.0972 & 0.0210 & 0.0873 & 0.0198 & 0.0994 & 0.0267 & 0.0559 & 0.0152 \\
\hline 30 & 1.46 & 0.24 & 0.152 & 0.027 & 0.609 & 0.115 & 0.196 & 0.038 & 0.134 & 0.028 & 0.125 & 0.027 & 0.103 & 0.023 & 0.0953 & 0.0256 & 0.0448 & 0.0122 \\
\hline 85 & 1.39 & 0.22 & 0.142 & 0.025 & 0.614 & 0.116 & 0.198 & 0.039 & 0.140 & 0.029 & 0.107 & 0.023 & 0.0661 & 0.0150 & 0.0833 & 0.0224 & 0.0381 & 0.0104 \\
\hline 0 & 1.32 & 0.21 & 0.166 & 0.029 & 0.506 & 0.095 & 0.167 & 0.032 & 0.149 & 0.031 & 0.128 & 0.028 & 0.0751 & 0.0170 & 0.0939 & 0.0252 & 0.0408 & 0.0111 \\
\hline 5 & 1.30 & 0.21 & 0.149 & 0.026 & 0.500 & 0.094 & 0.170 & 0.033 & 0.169 & 0.035 & 0.117 & 0.025 & 0.0637 & 0.0145 & 0.0943 & 0.0253 & 0.0413 & 0.0113 \\
\hline 100 & 1.31 & 0.21 & 0.151 & 0.027 & 0.537 & 0.101 & 0.138 & 0.027 & 0.160 & 0.033 & 0.120 & 0.026 & 0.0781 & 0.0177 & 0.0835 & 0.0224 & 0.0390 & 0.0106 \\
\hline 105 & 1.27 & 0.20 & 0.183 & 0.032 & 0.469 & 0.088 & 0.125 & 0.024 & 0.173 & 0.036 & 0.134 & 0.029 & 0.0607 & 0.0138 & 0.0880 & 0.0237 & 0.0322 & 0.0088 \\
\hline 110 & 1.27 & 0.20 & 0.169 & 0.030 & 0.452 & 0.085 & 0.127 & 0.025 & 0.148 & 0.031 & 0.168 & 0.036 & 0.0705 & 0.0160 & 0.0983 & 0.0264 & 0.0348 & 0.0095 \\
\hline 115 & 1.29 & 0.21 & 0.190 & 0.034 & 0.446 & 0.084 & 0.121 & 0.023 & 0.153 & 0.032 & 0.169 & 0.036 & 0.0578 & 0.0131 & 0.113 & 0.030 & 0.0400 & 0.0109 \\
\hline 120 & 1.30 & 0.21 & 0.251 & 0.044 & 0.436 & 0.082 & 0.122 & 0.024 & 0.142 & 0.030 & 0.161 & 0.035 & 0.0461 & 0.0105 & 0.0980 & 0.0263 & 0.0432 & 0.0118 \\
\hline 125 & 1.31 & 0.21 & 0.269 & 0.047 & 0.447 & 0.084 & 0.118 & 0.023 & 0.135 & 0.028 & 0.151 & 0.033 & 0.0503 & 0.0114 & 0.0940 & 0.0253 & 0.0493 & 0.0134 \\
\hline 130 & 1.33 & 0.21 & 0.296 & 0.052 & 0.475 & 0.090 & 0.120 & 0.023 & 0.123 & 0.026 & 0.135 & 0.029 & 0.0465 & 0.0106 & 0.0832 & 0.0224 & 0.0541 & 0.0147 \\
\hline $\begin{array}{l}\text { Fractional } \\
\text { error }\end{array}$ & & 0.161 & & 0.196 & & 0.205 & & 0.224 & & 0.233 & & 0.237 & & 0.263 & & 0.278 & & 0.273 \\
\hline
\end{tabular}

\begin{tabular}{|c|c|c|c|c|c|c|c|c|c|c|c|c|c|c|c|c|c|}
\hline 96.2 & 15.8 & 5.10 & 0.98 & 36.7 & 7.4 & 18.8 & 3.9 & 5.91 & 1.24 & 9.97 & 2.17 & 16.3 & 3.7 & 1.88 & 0.48 & 1.69 & 0.46 \\
\hline 89.9 & 14.7 & 4.98 & 0.95 & 34.1 & 6.9 & 17.6 & 3.6 & 5.14 & 1.08 & 9.38 & 2.04 & 15.5 & 3.5 & 1.83 & 0.47 & 1.32 & 0.36 \\
\hline 86.0 & 14.1 & 4.37 & 0.84 & 34.2 & 6.9 & 14.9 & 3.1 & 4.27 & 0.89 & 7.81 & 1.70 & 17.1 & 3.9 & 1.78 & 0.45 & 1.49 & 0.41 \\
\hline 77.8 & 12.7 & 4.01 & 0.77 & 28.2 & 5.7 & 15.7 & 3.2 & 3.78 & 0.79 & 8.40 & 1.83 & 14.9 & 3.4 & 1.66 & 0.42 & 1.26 & 0.34 \\
\hline 72.7 & 11.9 & 3.24 & 0.62 & 29.2 & 5.9 & 12.4 & 2.6 & 3.52 & 0.74 & 6.45 & 1.41 & 14.9 & 3.4 & 1.57 & 0.40 & 1.44 & 0.39 \\
\hline 60.2 & 9.9 & 2.38 & 0.46 & 25.9 & 5.2 & 10.5 & 2.2 & 3.09 & 0.65 & 4.65 & 1.02 & 11.3 & 2.6 & 1.36 & 0.35 & 1.08 & 0.29 \\
\hline 52.1 & 8.5 & 1.65 & 0.32 & 22.0 & 4.4 & 9.75 & 2.01 & 2.60 & 0.55 & 4.25 & 0.93 & 9.40 & 2.15 & 1.27 & 0.32 & 1.13 & 0.31 \\
\hline 42.7 & 7.0 & 1.03 & 0.20 & 19.3 & 3.9 & 7.38 & 1.52 & 2.07 & 0.44 & 3.31 & 0.72 & 7.70 & 1.76 & 1.06 & 0.27 & 0.837 & 0.228 \\
\hline 33.8 & 5.5 & 0.502 & 0.096 & 14.9 & 3.0 & 7.52 & 1.55 & 2.41 & 0.51 & 3.05 & 0.67 & 3.89 & 0.89 & 0.862 & 0.220 & 0.701 & 0.191 \\
\hline 24.5 & 4.0 & 0.257 & 0.049 & 11.8 & 2.4 & 4.93 & 1.02 & 1.65 & 0.35 & 2.33 & 0.51 & 2.41 & 0.55 & 0.650 & 0.166 & 0.503 & 0.137 \\
\hline 20.5 & 3.4 & 0.178 & 0.034 & 10.5 & 2.1 & 3.82 & 0.79 & 1.50 & 0.31 & 1.63 & 0.36 & 1.85 & 0.42 & 0.573 & 0.146 & 0.415 & 0.113 \\
\hline 11.6 & 1.9 & 0.238 & 0.045 & 5.82 & 1.18 & 2.10 & 0.43 & 1.13 & 0.24 & 0.991 & 0.216 & 0.722 & 0.165 & 0.336 & 0.086 & 0.269 & 0.073 \\
\hline 9.99 & 1.64 & 0.287 & 0.055 & 5.14 & 1.04 & 1.84 & 0.38 & 1.01 & 0.21 & 0.566 & 0.123 & 0.602 & 0.138 & 0.309 & 0.079 & 0.227 & 0.062 \\
\hline 7.48 & 1.23 & 0.259 & 0.050 & 3.85 & 0.78 & 1.32 & 0.27 & 0.732 & 0.154 & 0.456 & 0.099 & 0.420 & 0.096 & 0.249 & 0.063 & 0.191 & 0.052 \\
\hline 5.23 & 0.86 & 0.305 & 0.058 & 2.42 & 0.49 & 0.936 & 0.193 & 0.482 & 0.101 & 0.307 & 0.067 & 0.382 & 0.087 & 0.222 & 0.057 & 0.174 & 0.047 \\
\hline 4.86 & 0.80 & 0.273 & 0.052 & 2.32 & 0.47 & 0.865 & 0.178 & 0.377 & 0.079 & 0.241 & 0.053 & 0.383 & 0.088 & 0.224 & 0.057 & 0.172 & 0.047 \\
\hline 3.98 & 0.65 & 0.294 & 0.056 & 1.62 & 0.33 & 0.705 & 0.145 & 0.345 & 0.072 & 0.236 & 0.052 & 0.403 & 0.092 & 0.218 & 0.056 & 0.163 & 0.044 \\
\hline 3.48 & 0.57 & 0.258 & 0.049 & 1.47 & 0.30 & 0.559 & 0.115 & 0.219 & 0.046 & 0.249 & 0.054 & 0.386 & 0.088 & 0.198 & 0.050 & 0.141 & 0.038 \\
\hline 3.12 & 0.51 & 0.240 & 0.046 & 1.22 & 0.25 & 0.602 & 0.124 & 0.214 & 0.045 & 0.236 & 0.051 & 0.329 & 0.075 & 0.193 & 0.049 & 0.0845 & 0.0230 \\
\hline 2.64 & 0.43 & 0.204 & 0.039 & 1.13 & 0.23 & 0.467 & 0.096 & 0.161 & 0.034 & 0.204 & 0.044 & 0.258 & 0.059 & 0.124 & 0.032 & 0.0965 & 0.0262 \\
\hline 2.33 & 0.38 & 0.178 & 0.034 & 0.988 & 0.199 & 0.414 & 0.085 & 0.145 & 0.030 & 0.177 & 0.039 & 0.249 & 0.057 & 0.105 & 0.027 & 0.0775 & 0.0211 \\
\hline 2.11 & 0.34 & 0.175 & 0.033 & 0.885 & 0.179 & 0.327 & 0.067 & 0.156 & 0.033 & 0.147 & 0.032 & 0.267 & 0.061 & 0.0837 & 0.0213 & 0.0659 & 0.0179 \\
\hline 2.04 & 0.33 & 0.142 & 0.027 & 0.948 & 0.191 & 0.312 & 0.064 & 0.130 & 0.027 & 0.145 & 0.032 & 0.204 & 0.047 & 0.0910 & 0.0232 & 0.0703 & 0.0191 \\
\hline 1.88 & 0.31 & 0.130 & 0.025 & 0.887 & 0.179 & 0.269 & 0.055 & 0.134 & 0.028 & 0.131 & 0.029 & 0.175 & 0.040 & 0.0897 & 0.0228 & 0.0646 & 0.0176 \\
\hline 1.84 & 0.30 & 0.133 & 0.026 & 0.951 & 0.192 & 0.229 & 0.047 & 0.130 & 0.027 & 0.0946 & 0.0206 & 0.135 & 0.031 & 0.0971 & 0.0247 & 0.0680 & 0.0185 \\
\hline 1.77 & 0.29 & 0.119 & 0.023 & 0.919 & 0.186 & 0.208 & 0.043 & 0.116 & 0.024 & 0.108 & 0.024 & 0.123 & 0.028 & 0.100 & 0.025 & 0.0742 & 0.0202 \\
\hline 1.58 & 0.26 & 0.126 & 0.024 & 0.766 & 0.155 & 0.171 & 0.035 & 0.133 & 0.028 & 0.0908 & 0.0198 & 0.137 & 0.031 & 0.0891 & 0.0227 & 0.0667 & 0.0181 \\
\hline 1.61 & 0.26 & 0.142 & 0.027 & 0.744 & 0.150 & 0.178 & 0.037 & 0.135 & 0.028 & 0.0872 & 0.0190 & 0.129 & 0.029 & 0.112 & 0.028 & 0.0816 & 0.0222 \\
\hline 1.61 & 0.26 & 0.171 & 0.033 & 0.738 & 0.149 & 0.197 & 0.041 & 0.147 & 0.031 & 0.0565 & 0.0123 & 0.115 & 0.026 & 0.113 & 0.029 & 0.0688 & 0.0187 \\
\hline 1.57 & 0.26 & 0.189 & 0.036 & 0.619 & 0.125 & 0.195 & 0.040 & 0.175 & 0.037 & 0.0583 & 0.0127 & 0.159 & 0.036 & 0.0974 & 0.0248 & 0.0748 & 0.0203 \\
\hline 1.50 & 0.25 & 0.205 & 0.039 & 0.545 & 0.110 & 0.181 & 0.037 & 0.156 & 0.033 & 0.0708 & 0.0154 & 0.160 & 0.037 & 0.106 & 0.027 & 0.0770 & 0.0209 \\
\hline 1.54 & 0.25 & 0.244 & 0.047 & 0.585 & 0.118 & 0.175 & 0.036 & 0.170 & 0.036 & 0.0687 & 0.0150 & 0.121 & 0.028 & 0.0961 & 0.0245 & 0.0767 & 0.0209 \\
\hline 1.69 & 0.28 & 0.299 & 0.057 & 0.587 & 0.118 & 0.207 & 0.043 & 0.134 & 0.028 & 0.108 & 0.024 & 0.170 & 0.039 & 0.113 & 0.029 & 0.0714 & 0.0194 \\
\hline 1.70 & 0.28 & 0.351 & 0.067 & 0.544 & 0.110 & 0.196 & 0.040 & 0.156 & 0.033 & 0.120 & 0.026 & 0.186 & 0.043 & 0.0941 & 0.0240 & 0.0566 & 0.0154 \\
\hline 1.78 & 0.29 & 0.369 & 0.071 & 0.568 & 0.115 & 0.208 & 0.043 & 0.180 & 0.033 & 0.154 & 0.034 & 0.152 & 0.035 & 0.0964 & 0.0246 & 0.0483 & 0.0131 \\
\hline
\end{tabular}


TABLE III. (Continued.)

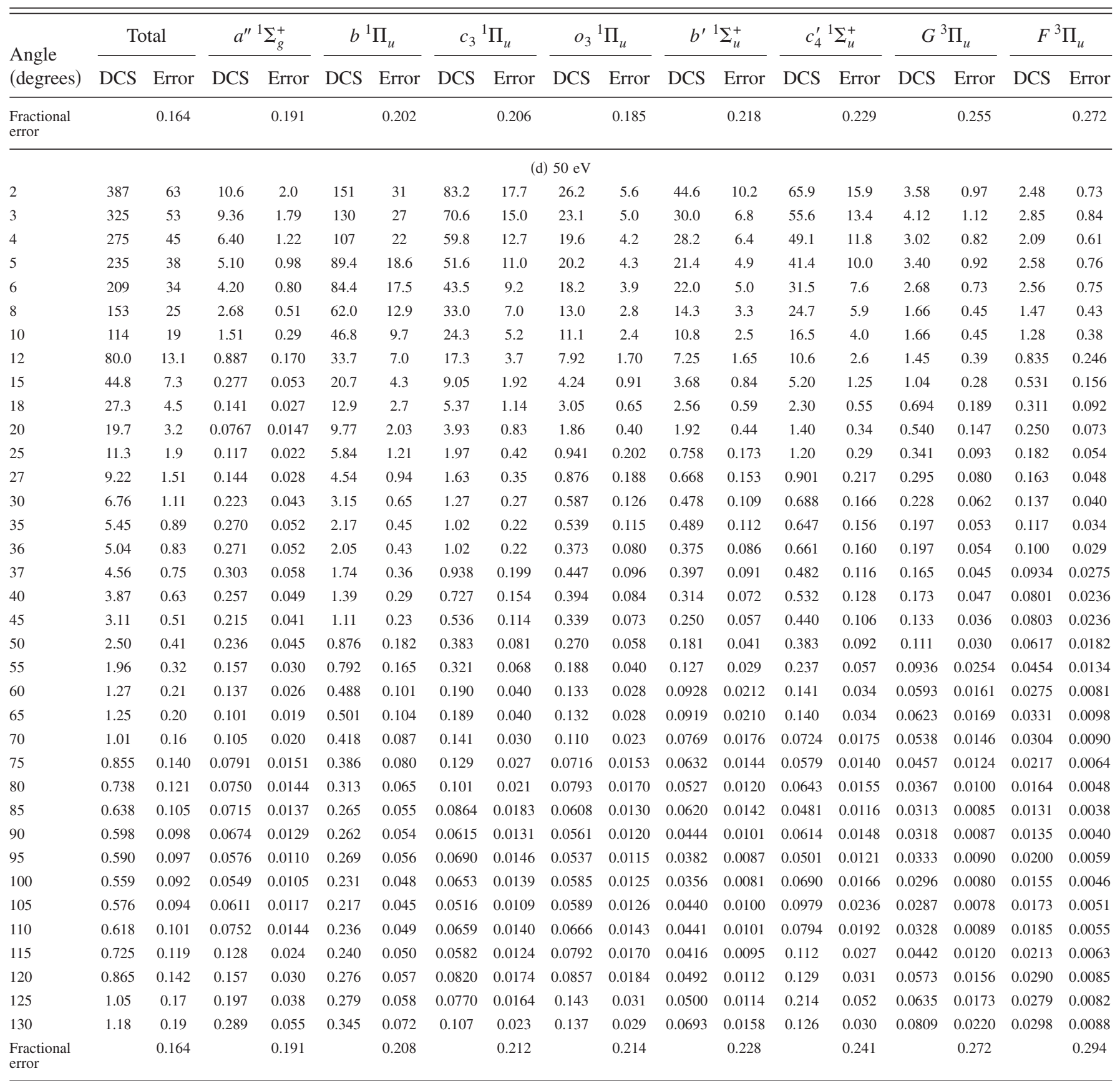

(e) $100 \mathrm{eV}$

\begin{tabular}{|c|c|c|c|c|c|c|c|c|c|c|c|c|c|c|c|c|c|c|}
\hline & 1002 & 166 & 13.2 & 2.7 & 374 & 77 & 223 & 47 & 103 & 21 & 90.0 & 21.0 & 194 & 47 & 3.98 & 1.21 & 1.65 & 0.55 \\
\hline 3 & 747 & 124 & 9.57 & 1.94 & 280 & 58 & 160 & 34 & 82.9 & 17.3 & 60.0 & 14.0 & 148 & 36 & 4.11 & 1.25 & 2.43 & 0.81 \\
\hline & 557 & 93 & 8.00 & 1.63 & 220 & 45 & 115 & 24 & 62.2 & 13.0 & 46.1 & 10.8 & 100 & 25 & 3.51 & 1.07 & 2.28 & 0.76 \\
\hline & 378 & 63 & 4.86 & 0.99 & 146 & 30 & 75.8 & 16.0 & 39.0 & 8.1 & 28.0 & 6.5 & 79.4 & 19.5 & 3.42 & 1.04 & 2.14 & 0.71 \\
\hline & 284 & 47 & 3.59 & 0.73 & 109 & 22 & 56.9 & 12.0 & 31.5 & 6.6 & 19.7 & 4.6 & 58.3 & 14.3 & 2.92 & 0.89 & 2.27 & 0.75 \\
\hline & 159 & 26 & 2.25 & 0.46 & 64.6 & 13.4 & 32.2 & 6.8 & 16.5 & 3.4 & 10.8 & 2.5 & 29.3 & 7.2 & 1.74 & 0.53 & 1.48 & 0.49 \\
\hline 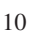 & 82.2 & 13.7 & 1.06 & 0.22 & 33.5 & 6.9 & 16.1 & 3.4 & 9.62 & 2.00 & 4.91 & 1.15 & 15.3 & 3.8 & 0.838 & 0.255 & 0.869 & 0.289 \\
\hline & 42.3 & 7.0 & 0.552 & 0.112 & 19.9 & 4.1 & 8.30 & 1.75 & 4.25 & 0.89 & 2.79 & 0.65 & 5.86 & 1.44 & 0.380 & 0.116 & 0.282 & 0.094 \\
\hline 5 & 20.4 & 3.4 & 0.239 & 0.049 & 9.87 & 2.04 & 3.67 & 0.77 & 2.14 & 0.45 & 1.39 & 0.32 & 2.64 & 0.65 & 0.236 & 0.072 & 0.163 & 0.054 \\
\hline & 11.4 & 1.9 & 0.145 & 0.030 & 6.00 & 1.24 & 1.89 & 0.40 & 1.18 & 0.25 & 0.726 & 0.170 & 1.24 & 0.30 & 0.146 & 0.045 & 0.0910 & 0.0303 \\
\hline 20 & 8.59 & 1.43 & 0.181 & 0.037 & 4.25 & 0.88 & 1.29 & 0.27 & 0.915 & 0.191 & 0.596 & 0.139 & 1.14 & 0.28 & 0.133 & 0.040 & 0.0803 & 0.0267 \\
\hline 25 & 5.52 & 0.92 & 0.330 & 0.067 & 2.26 & 0.47 & 0.751 & 0.158 & 0.645 & 0.134 & 0.395 & 0.092 & 1.01 & 0.25 & 0.0892 & 0.0271 & 0.0455 & 0.0151 \\
\hline 1 & 4.82 & 0.80 & 0.374 & 0.076 & 1.64 & 0.34 & 0.826 & 0.174 & 0.630 & 0.131 & 0.375 & 0.088 & 0.851 & 0.209 & 0.0815 & 0.0248 & 0.0372 & 0.0124 \\
\hline
\end{tabular}


TABLE III. (Continued.)

\begin{tabular}{|c|c|c|c|c|c|c|c|c|c|c|c|c|c|c|c|c|c|c|}
\hline \multirow{2}{*}{$\begin{array}{l}\text { Angle } \\
\text { (degrees) }\end{array}$} & \multicolumn{2}{|c|}{ Total } & \multicolumn{2}{|c|}{$a^{\prime \prime}{ }^{1} \Sigma_{g}^{+}$} & \multicolumn{2}{|c|}{$b^{1} \Pi_{u}$} & \multicolumn{2}{|c|}{$c_{3}{ }^{1} \Pi_{u}$} & \multicolumn{2}{|c|}{$o_{3}{ }^{1} \Pi_{u}$} & \multicolumn{2}{|c|}{$b^{\prime}{ }^{1} \Sigma_{u}^{+}$} & \multicolumn{2}{|c|}{$c_{4}^{\prime}{ }^{1} \Sigma_{u}^{+}$} & \multicolumn{2}{|c|}{$G^{3} \Pi_{u}$} & \multicolumn{2}{|c|}{$F^{3} \Pi_{u}$} \\
\hline & DCS & Error & DCS & Error & DCS & Error & DCS & Error & DCS & Error & DCS & Error & DCS & Error & DCS & Error & DCS & Error \\
\hline 30 & 3.88 & 0.65 & 0.363 & 0.074 & 1.36 & 0.28 & 0.675 & 0.142 & 0.487 & 0.102 & 0.315 & 0.074 & 0.579 & 0.142 & 0.0660 & 0.0200 & 0.0349 & 0.0116 \\
\hline 35 & 2.52 & 0.42 & 0.329 & 0.067 & 0.784 & 0.162 & 0.438 & 0.092 & 0.347 & 0.072 & 0.212 & 0.049 & 0.324 & 0.079 & 0.0462 & 0.0140 & 0.0376 & 0.0125 \\
\hline 37 & 2.03 & 0.34 & 0.257 & 0.052 & 0.692 & 0.143 & 0.300 & 0.063 & 0.269 & 0.056 & 0.126 & 0.030 & 0.327 & 0.080 & 0.0392 & 0.0119 & 0.0243 & 0.0081 \\
\hline 40 & 1.59 & 0.26 & 0.202 & 0.041 & 0.529 & 0.109 & 0.245 & 0.052 & 0.202 & 0.042 & 0.147 & 0.034 & 0.209 & 0.051 & 0.0336 & 0.0102 & 0.0239 & 0.0080 \\
\hline 45 & 1.05 & 0.17 & 0.145 & 0.029 & 0.340 & 0.070 & 0.178 & 0.038 & 0.129 & 0.027 & 0.106 & 0.025 & 0.112 & 0.028 & 0.0210 & 0.0064 & 0.0174 & 0.0058 \\
\hline 50 & 0.687 & 0.114 & 0.111 & 0.022 & 0.235 & 0.049 & 0.0677 & 0.0143 & 0.0960 & 0.0200 & 0.0513 & 0.0120 & 0.0954 & 0.0234 & 0.0204 & 0.0062 & 0.0102 & 0.0034 \\
\hline 55 & 0.543 & 0.090 & 0.0861 & 0.0175 & 0.199 & 0.041 & 0.0793 & 0.0167 & 0.0580 & 0.0121 & 0.0251 & 0.0059 & 0.0630 & 0.0155 & 0.0226 & 0.0069 & 0.00988 & 0.00329 \\
\hline $\begin{array}{l}\text { Fractional } \\
\text { error }\end{array}$ & & 0.166 & & 0.203 & & 0.207 & & 0.211 & & 0.208 & & 0.233 & & 0.245 & & 0.304 & & 0.333 \\
\hline
\end{tabular}

\section{RESULTS AND DISCUSSION}

Table III displays the DCSs for the electron-impact excitation of the transitions measured in this work. The average uncertainties are also given in this table. Unfortunately, the intensities of the $D^{3} \Sigma_{u}^{+}$state $v^{\prime}$ levels were too small to generate reliable DCSs and consequently no $D^{3} \Sigma_{u}^{+}$state DCSs are reported in Table III. However, an upper limit on the $D^{3} \Sigma_{u}^{+}$state cross sections can be estimated to be approximately an order of magnitude smaller than the smallest measured DCS-i.e., on the order of $10^{-21} \mathrm{~cm}^{2} \mathrm{sr}^{-1}$. In comparison with earlier energy-loss work, we find this result is similar to that observed by Chutjian et al. [26] (renormalized by Trajmar et al. [19]); i.e., they also did not observe any significant scattering intensity for the $D^{3} \Sigma_{u}^{+}$state excitation at the studied incident energies. Of note, in Table I, nonzero REPs were reported for the $D(0)$ and $D(1)$ features based on the minimal intensity attributed to these levels (see Sec. II B.). Since no intensity was attributable to the $D(2)$ or $D(3)$ levels, we reported zero-valued REPs for the $D(2)$ and $D(3)$ levels. Assuming the excitation probabilities of $D\left(v^{\prime}>3\right)$ levels are negligible (based on RKR FC calculations), the REPs assigned to the $D(0)$ and $D(1)$ levels were normalized such that they summed to unity. We now address the DCSs for the individual excitations.

\section{A. Excitation differential cross sections}

\section{The $a^{\prime \prime}{ }^{1} \Sigma_{g}^{+}$excitation}

Figure 3 shows our DCSs for the $X^{1} \Sigma_{g}^{+}\left(v^{\prime \prime}=0\right)$ $\rightarrow a^{\prime \prime}{ }^{1} \Sigma_{g}^{+}\left(v^{\prime}=0,1\right)$ excitation, with the results compared to earlier measurements, where possible. From Fig. 3(a) $\left(E_{0}\right.$ $=17.5 \mathrm{eV}$ ), we observe that our DCS is approximately a factor of 2 lower than the measurements of Trajmar et al. [19] (at $E_{0}=17 \mathrm{eV}$ ), Brunger and Teubner [20], and Zubek and King [23]. Applying Eq. (1) at this energy generated approximately a $<5 \%$ correction. Our DCSs show a sharp drop at $\theta<8^{\circ}$, which is not unexpected for a forbidden $\Sigma \leftrightarrow \Sigma$ transition. In addition, the shape of our DCSs at $\theta>80^{\circ}$ differs markedly from the other results. We also find a small inflection in the DCS trend at $\theta \approx 30^{\circ}$. The systematic difference from the earlier measurements is likely due to differences in the transmission of the instruments [56]. However, we note that our DCSs have the advantage of being corrected by the TOF results of [43] in conjunction with the movable source method for elastic background subtraction [42] and, subsequently, are probably systematically more accurate. In Fig. 3(b) $\left(E_{0}=20 \mathrm{eV}\right)$, the disagreement by a factor of $\sim 2$ still remains, but the DCS shapes at $\theta>80^{\circ}$ show improved agreement with the earlier work. Because of the coarser angular steps used in the earlier measurements, they appear to have overstepped the more developed minimum in the $a^{\prime \prime}{ }^{1} \Sigma_{g}^{+}$DCS at $\theta \approx 20^{\circ}$. In Fig. 3(c) $\left(E_{0}=30 \mathrm{eV}\right)$, our DCS is in better agreement with the DCSs of Trajmar et al. [19], except for their downward trend for $\theta>80^{\circ}$, which opposes their trend at the lower $E_{0}$ value of $20 \mathrm{eV}$. Qualitative agreement with Brunger and Teubner's [20] DCS is better, but their results are still higher than ours, by about $50 \%$. We note the fully developed "cusplike" feature at $\theta=20^{\circ}$ in the $a^{\prime \prime}{ }^{1} \Sigma_{g}^{+}$ DCS measured in the present work. At $E_{0}=50 \mathrm{eV}[$ Fig. 3(d)], agreement between the various DCS measurements is very good, except for the decreasing DCS of [19] at $\theta>110^{\circ}$. In fact, in this region, we observe a steep rise, with our DCS larger than that of [19] by over a factor of 4 in the high- $\theta$ limit. At this $E_{0}$ value, measurements of both $[19,20]$ seem to be consistent with the presence of the cusp, but do not probe it because of the coarser angular spacing of their work. The cusp is clearly observed in the present work at $\theta=20^{\circ}$, with an angular width of about $9^{\circ}$. At $E_{0}=100 \mathrm{eV}$ [Fig. 3(e)], the cusp still persists.

This cusp is interesting because it is likely due to channelcoupling effects, similar to the cusps observed in atomic transitions where the lower and upper states have the same symmetry-e.g., the $1{ }^{1} S$ to $2{ }^{1} S$ transition in $\mathrm{He}$ - and has been discussed in detail in an earlier paper by our group [12]. It is probably not as pronounced in molecules as it is atoms, on account of being washed out by alignment and rotational effects in the molecule that are not present in atoms. It would be of interest for a theoretical investigation to look at this transition in the fixed-nuclei approximation to see how pronounced this cusp is and adding rotation to see how this may affect the depth of this feature. This is the first time to our knowledge (see [12]) that such a cusp, in differential scattering of electrons from molecular targets, has been distinctly observed.

The disagreement between our DCSs and those of Brunger and Teubner [20] for this transition may also explain 

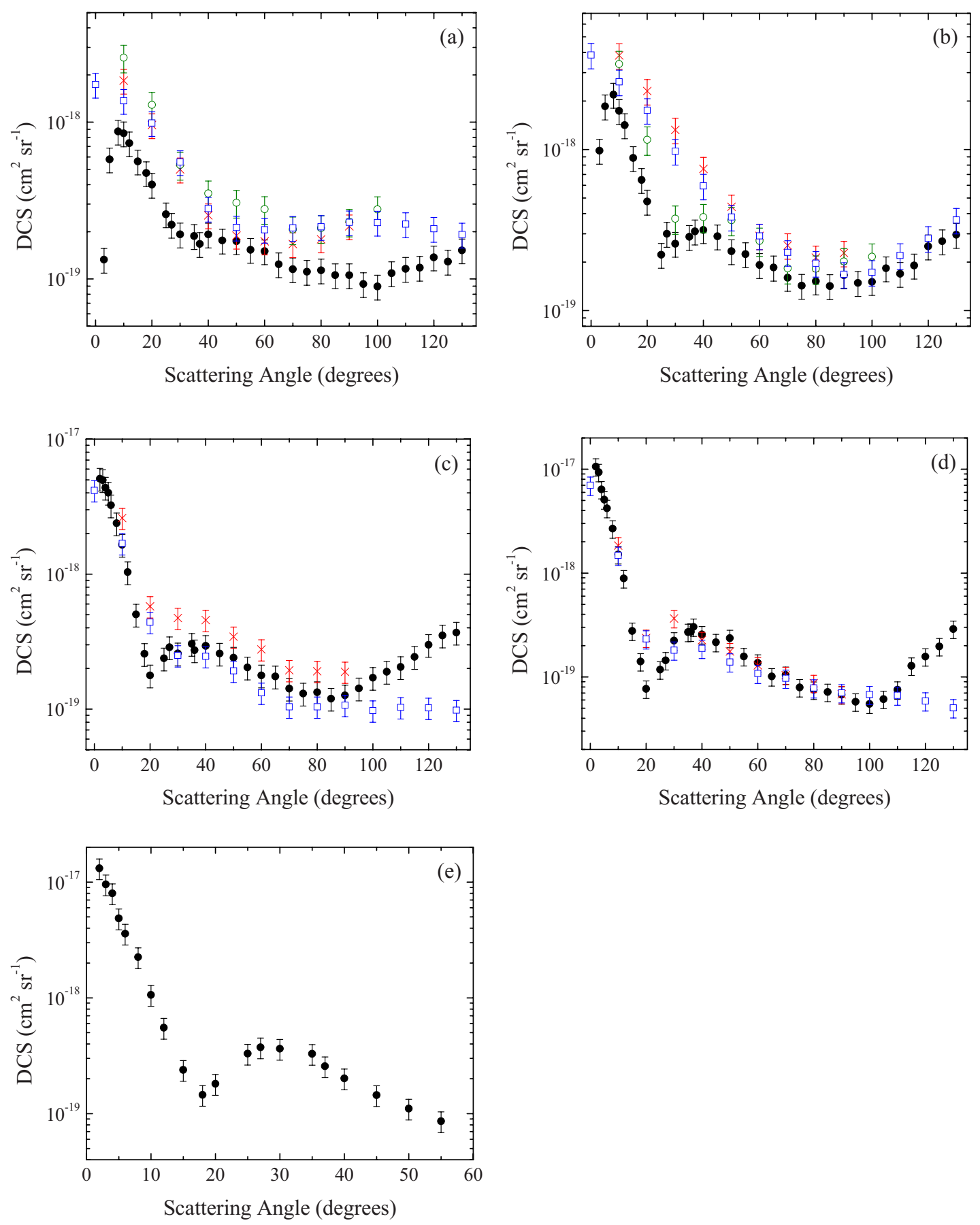

FIG. 3. (Color online) DCSs for the electron-impact excitations to the $a^{\prime \prime}{ }^{1} \Sigma_{g}^{+}$state with the following $E_{0}$ : (a) $17.5 \mathrm{eV}$, (b) $20 \mathrm{eV}$, (c) $30 \mathrm{eV}$, (d) $50 \mathrm{eV}$, and (e) $100 \mathrm{eV}$ (reproduced from [12] with permission from the Institute of Physics Publishing). Legend: $\bullet$, present work; $\square$, Trajmar et al. [19] (at $E_{0}=17 \mathrm{eV}$ ); $\times$, Brunger and Teubner [20]; $\bigcirc$, Zubek and King [23].

the disagreements between our DCSs for the lower transitions in $\mathrm{N}_{2}$ published in [1], since Brunger and Teubner used the scattering intensity of the $a^{\prime \prime}{ }^{1} \Sigma_{g}^{+}$state as a calibration check in their electron-scattering measurements (see [20]). Furthermore, the DCS cusp in this excitation will affect the overall total excitation cross-section determinations (i.e., ICSs from the DCSs) on the order of $\sim 15 \%$ and may partially account for the discrepancies between the ICSs of $[19,57,58]$, where the ICSs of $[57,58]$ were derived from the DCSs of $[20,1]$, respectively. However, apparent backscatter- ing observed in the present work may counter the influence of the cusp structure on the derived ICSs. This will be considered in detail in a future paper.

We also note here that, from our measurements, the $v^{\prime}$ $=0$ level of the $a^{\prime \prime}{ }^{1} \Sigma_{g}^{+}$state dominates this excitation, accounting for $\approx 85 \%$ of the total intensity (see Table I). Interestingly, at large scattering angles $\left(\theta>70^{\circ}\right)$, the $a^{\prime \prime}{ }^{1} \Sigma_{g}^{+}\left(v^{\prime}\right.$ $=1$ ) feature becomes broadened. We found that we could best compensate for this "broadening" by adding a state at the energy-loss value of $12.540 \mathrm{eV}$ (see $M$ in Table I). If $M$ 

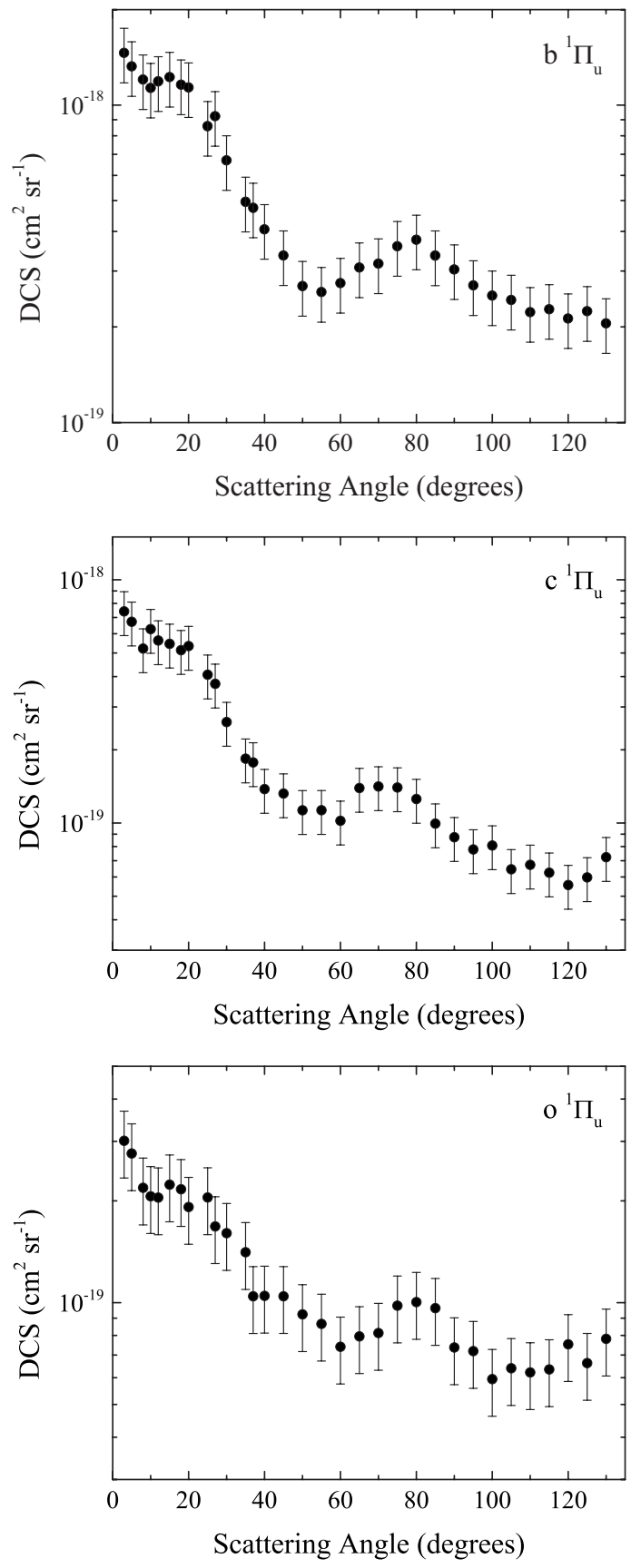

FIG. 4. DCSs for the electron-impact excitations to the $b{ }^{1} \Pi_{u}$, $c_{3}{ }^{1} \Pi_{u}$, and $o_{3}{ }^{1} \Pi_{u}$ states at $E_{0}=17.5 \mathrm{eV}$. Legend: $\bullet$, present work. See text for details regarding vibrational coverage in the last paragraph of Sec. II B.

is not included at large angles, the experimental FC factors (present REPs), linearly averaged over the $70^{\circ}-130^{\circ}$ range, give values of 0.89 and 0.11 , for the $v^{\prime}=0$ and 1 levels of the $a^{\prime \prime}{ }^{1} \Sigma_{g}^{+}$state, respectively, thus inflating the relative $a^{\prime \prime}{ }^{1} \Sigma_{g}^{q}\left(v^{\prime}=0\right)$ contribution. Therefore, the contribution from the $M$ feature is included in the DCSs for the $a^{\prime \prime}{ }^{1} \Sigma_{g}^{+}$state. The source of the broadening is not presently understood. The circumstantial evidence presented in [59] is noteworthy due to the suggestion of predissociation of the $a^{\prime \prime}{ }^{1} \Sigma_{g}^{+}$state, which could be involved with the observed broadening. However, the resulting $3.5 \mu$ s lifetime of the $v^{\prime}=0$ level
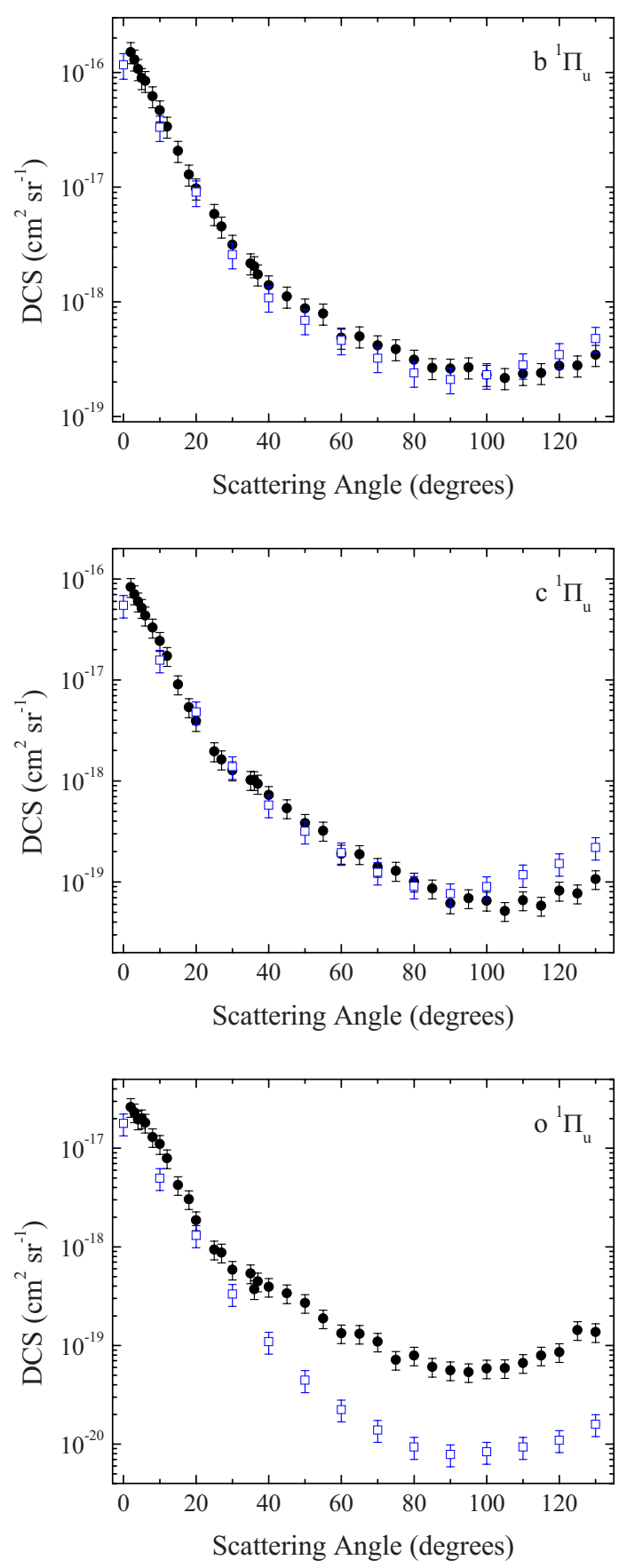

FIG. 5. (Color online) DCSs for the electron-impact excitations to the $b{ }^{1} \Pi_{u}, c_{3}{ }^{1} \Pi_{u}$, and $o_{3}{ }^{1} \Pi_{u}$ states at $E_{0}=50 \mathrm{eV}$. Legend: $\bullet$, present work; $\square$, Trajmar et al. [19], average of their $E_{0}=40$ and $60 \mathrm{eV}$ DCSs. See text for details regarding vibrational coverage in the last paragraph of Sec. II B.

should be a negligible factor in direct energy-loss measurements due to the time scale of the electron-scattering events, though this could have a slightly more significant influence on backscattered electrons. No broadening of the $v^{\prime}=0$ level was observed. However, the "broadening" of the $v^{\prime}=1$ level perhaps indicates excitation of a forbidden state not readily assignable at present. We hope that the work presented here and in [12] will stimulate theoretical and experimental stud- 

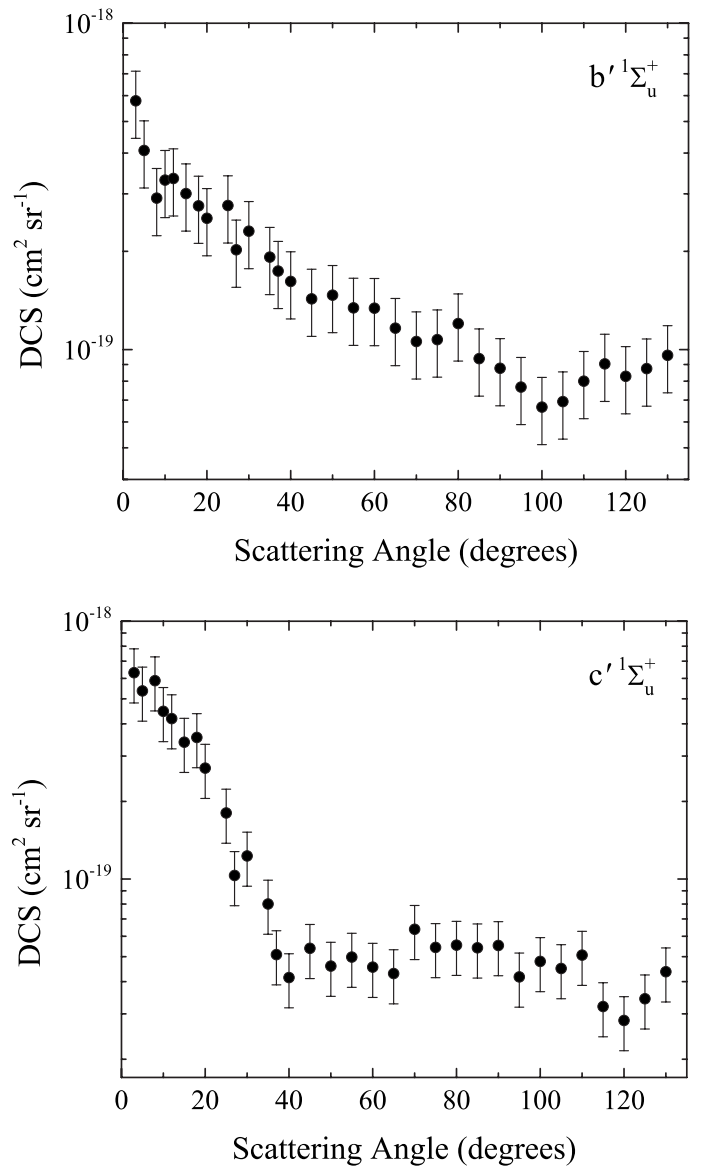

FIG. 6. DCSs for the electron-impact excitations to the $b^{\prime}{ }^{1} \Sigma_{u}^{+}$ and $c_{4}^{\prime}{ }^{1} \Sigma_{u}^{+}$states at $E_{0}=17.5 \mathrm{eV}$. Legend: $\bullet$, present work. See text for details regarding vibrational coverage in the last paragraph of Sec. II B.

ies to determine the underlying physics of this effect and that of the observed cusp structure.

\section{The $b^{1} \Pi_{w}, c_{3}{ }^{1} \Pi_{w}$, and $o_{3}{ }^{1} \Pi_{u}$ state excitations}

Figures 4 and 5 show the DCSs for excitation of the $b^{1} \Pi_{u}, c_{3}{ }^{1} \Pi_{u}$, and $o_{3}{ }^{1} \Pi_{u}$ states in the energy-loss interval of this work-i.e., summed over measured $v^{\prime}$-at representative $E_{0}$ values of 17.5 and $50 \mathrm{eV}$, respectively. From these figures, it is evident that all three excitations show similar angular patterns with rapid changes in the region of $\theta$ around $40^{\circ}-60^{\circ}$ at $E_{0}=17.5 \mathrm{eV}$. However, some (small) differences at larger scattering angles can be observed, especially for the $o_{3}{ }^{1} \Pi_{u}$ excitation. At $E_{0}=50 \mathrm{eV}$, these differences are almost negligible, typical of transitions to excited states of the same symmetry, especially in this case of strongly-mixed Rydberg and valence states. As expected, all DCSs show clear forward-scattering patterns typical of dipole-allowed transitions.

At $50 \mathrm{eV}$ (Fig. 5), we compare with the DCSs (with 25\% uncertainty) of Trajmar et al. [19] for these excitations. The DCSs of Trajmar et al. [19] were taken at $E_{0}=40$ and $60 \mathrm{eV}$, so for this comparison, an average of their two data sets was taken. The good agreement between the two data sets is surprising, considering that the earlier measurements used an
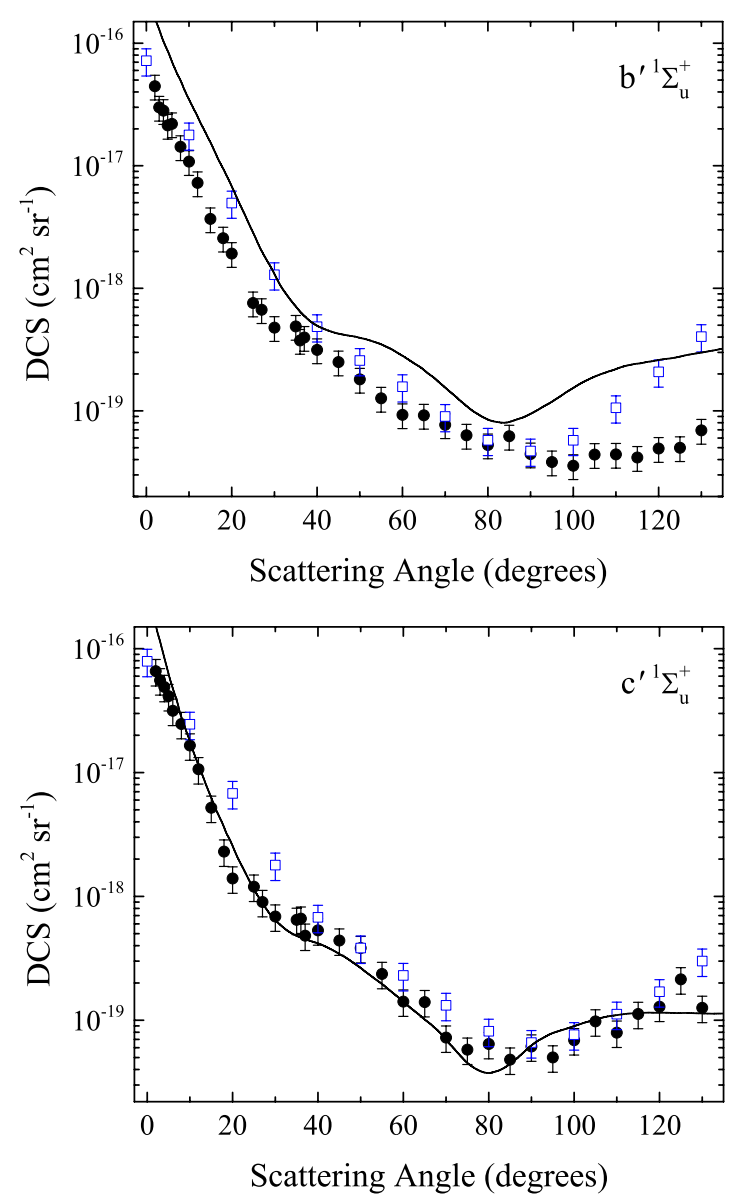

FIG. 7. (Color online) DCSs for the electron-impact excitations to the $b^{\prime}{ }^{1} \Sigma_{u}^{+}$and $c_{4}^{\prime}{ }^{1} \Sigma_{u}^{+}$states at $E_{0}=50 \mathrm{eV}$. Legend: $\bullet$, present work; $\square$, Trajmar et al. [19], average of their $E_{0}=40$ and $60 \mathrm{eV}$ DCSs; solid line, Mu-Tao and McKoy [32], average of their $E_{0}$ $=40$ and $60 \mathrm{eV}$ DCSs. See text for details regarding vibrational coverage in the last paragraph of Sec. II B.

FC factor algorithm to unfold their spectra. As has been pointed out in Sec. I, problems may arise in the fitting of an FC-factor-based algorithm to spectra acquired under different scattering conditions. Furthermore, there are some incorrect assignments included in the analysis of Chutjian et al. [26], who, indeed, acknowledged some uncertainty in them. In particular, of most relevance here, are their assignments of the $b(6)$ level to $G(1)$, and (part of) the $b(3)$ level to $F(0)$. These assignments may have helped to prevent distortions in their spectral fits, especially at high scattering angles in the energy-loss region containing strong quantum-interference effects [6] associated with interactions between the $b{ }^{1} \Pi_{u}$ and $c_{3}{ }^{1} \Pi_{u}$ states in the region of the $b{ }^{1} \Pi_{u}\left(v^{\prime}=5,6,7\right)$ and $c_{3}{ }^{1} \Pi_{u}\left(v^{\prime}=0,1\right)$ levels. One would have expected these interference effects to have resulted in a DCS for excitation to the $b{ }^{1} \Pi_{u}$ state, which is too low in the region where the $b(6)$ level feature reaches maximum relative intensity-i.e., at about $\theta=90^{\circ}$. In fact, in this region the two DCSs are in good agreement, allowing for an estimated uncertainty of (at least) $25 \%$ in the DCS of $[19,26]$. The overall agreement between the present DCSs and those of Trajmar et al. [19], for the $b^{1} \Pi_{u}$ and $c_{3}{ }^{1} \Pi_{u}$ states, is also surprisingly good. In the 

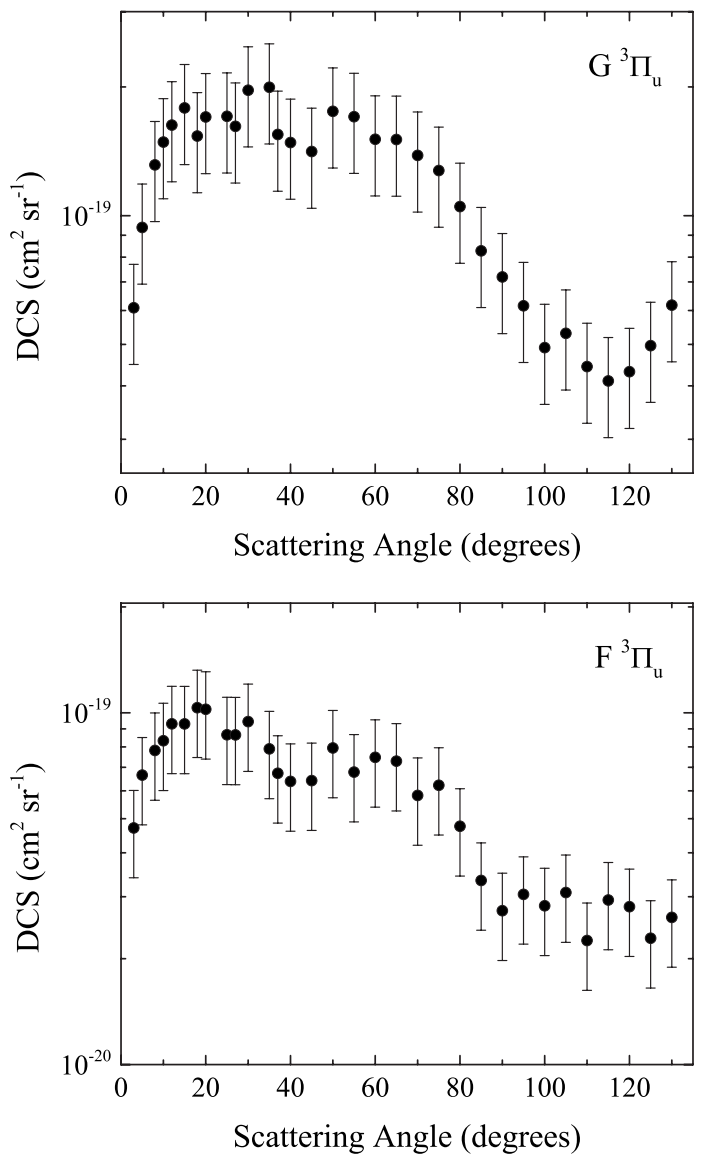

FIG. 8. DCSs for the electron-impact excitations to the $G^{3} \Pi_{u}$ and $F^{3} \Pi_{u}$ states at $E_{0}=17.5 \mathrm{eV}$. Legend: $\bullet$, present work. See text for details regarding vibrational coverage in the last paragraph of Sec. II B.

energy-loss range of this work, we capture $97.3 \%$ of the intensity for the $b{ }^{1} \Pi_{u}$ state, approximately $100 \%$ of the $c_{3}{ }^{1} \Pi_{u}$ state intensity, and $91.2 \%$ of the $o_{3}{ }^{1} \Pi_{u}$ state intensity, which is based on the coupled results of Stahel et al. [8]. Given this, the reason for the significant disagreement between our $o_{3}{ }^{1} \Pi_{u}$ state DCS and that of Trajmar et al. [19] is not clear. However, it should be noted that the $o_{3}(0)$ feature is very weak and all other $o_{3}{ }^{1} \Pi_{u}$ state $v^{\prime}$ levels are severely overlapped by features from other states, making their unfolding very sensitive to the particular FC factor assumptions of the data analysis. Overall, however, the agreement between the two sets of DCSs for the $b{ }^{1} \Pi_{u}, c_{3}{ }^{1} \Pi_{u}$, and $o_{3}{ }^{1} \Pi_{u}$ states is very encouraging, seeming to indicate that the vibrationally summed DCSs returned by the least-squares fitting procedures are not very sensitive to the details of the individual relative strengths, particularly if some of the stronger levels are "fully" resolved in the experimental spectra.

\section{The $b^{\prime}{ }^{1} \Sigma_{u}^{+}$and $c_{4}^{\prime}{ }^{1} \Sigma_{u}^{+}$state excitations}

Figures 6 and 7 show the DCSs of the $b^{\prime}{ }^{1} \Sigma_{u}^{+}$and $c_{4}^{\prime}{ }^{1} \Sigma_{u}^{+}$ states, for $E_{0}$ values of 17.5 and $50 \mathrm{eV}$. Results are found to be similar to those obtained for the $b{ }^{1} \Pi_{u}, c_{3}{ }^{1} \Pi_{u}$, and $o_{3}{ }^{1} \Pi_{u}$ states at these energies, with DCSs characteristic of dipole-
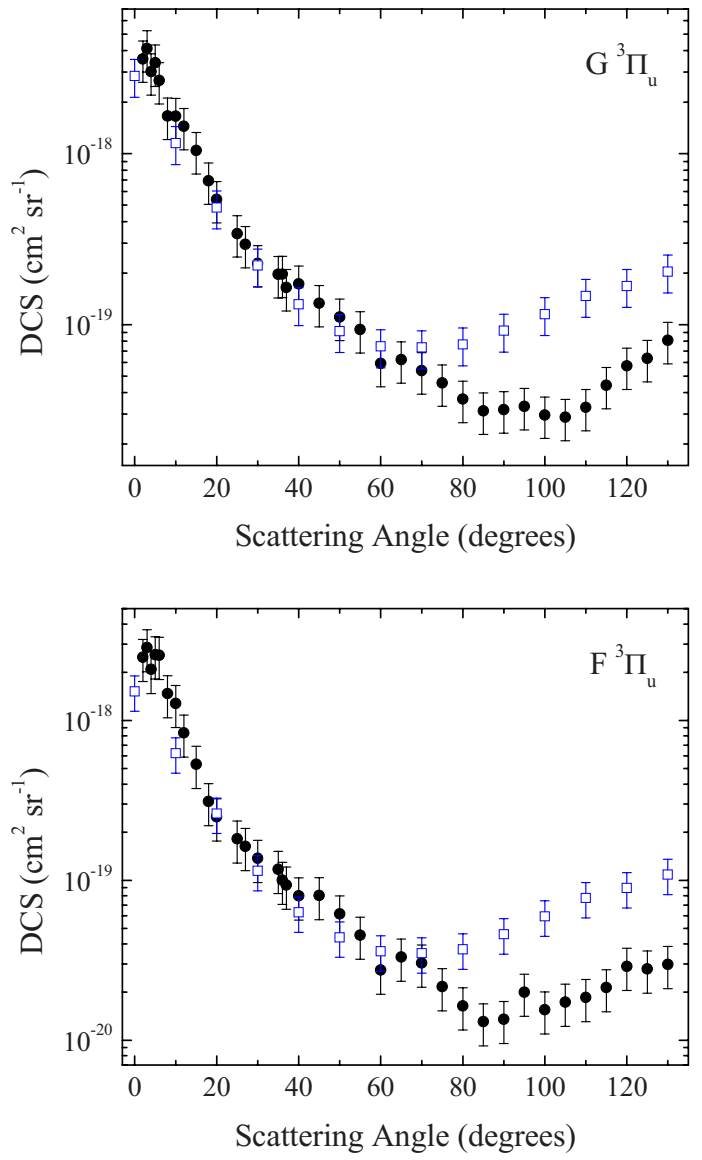

FIG. 9. (Color online) DCSs for the electron-impact excitations to the $G^{3} \Pi_{u}$ and $F^{3} \Pi_{u}$ states at $E_{0}=50 \mathrm{eV}$. Legend: present work; $\square$, Trajmar et al. [19], average of their $E_{0}=40$ and $60 \mathrm{eV}$. See text for details regarding vibrational coverage in the last paragraph of Sec. II B.

allowed transitions to states of a common symmetry, but with some variation in angular behavior at large $\theta$ values. Good agreement is found at $50 \mathrm{eV}$ with the DCSs (with $25 \%$ uncertainty) of Trajmar et al. [19] (where an average of the DCS at $E_{0}=40$ and $60 \mathrm{eV}$ were taken), especially for the $c_{4}^{\prime}{ }^{1} \Sigma_{u}^{+}$state, where remarkably close agreement in the shapes of the two data sets across the whole angular range is observed. In the energy-loss range of the present work $(12-13.82 \mathrm{eV})$, we capture only $8.1 \%$ of the intensity for the $b^{\prime}{ }^{1} \Sigma_{u}^{+}$state, while $82.5 \%$ of the $c_{4}^{\prime}{ }^{1} \Sigma_{u}^{+}$state intensity is observed. Thus, much of the $b^{\prime}{ }^{1} \Sigma_{u}^{+}$state intensity lies outside of the present work's energy-loss range. In the measurements of Trajmar et al. [19], with an energy-loss range extending to $14.2 \mathrm{eV}, \sim 40 \%$ of the $b^{\prime}{ }^{1} \Sigma_{u}^{+}$state spectrum was captured. Hence, the very good agreement between the two data sets is very surprising.

Mu-Tao and McKoy [32] provide DCSs using a distortedwave method for the $b^{\prime}{ }^{1} \Sigma_{u}^{+}$and $c_{4}^{\prime}{ }^{1} \Sigma_{u}^{+}$states at 40 and $60 \mathrm{eV}$, which are in good agreement with the Trajmar et al. [19] (Chutjian et al. [26]) data. The Mu-Tao and McKoy results have been digitized and averaged to give DCSs at $50 \mathrm{eV}$ using the same procedure applied to the DCSs of Trajmar et al. described in the preceding paragraph. The resulting 50-eV DCSs are plotted in Fig. 7. Excellent agreement is 
seen between the shapes of the distorted-wave and present $c_{4}^{\prime}{ }^{1} \Sigma_{u}^{+}$state DCSs with very good agreement in magnitude. We note that the vibrational contributions may partly explain the difference near zero degrees. The $b^{\prime}{ }^{1} \Sigma_{u}^{+}$state DCSs show reasonable agreement in magnitude considering the limited vibrational coverage, along with a consistent shape.

\section{The $G^{3} \Pi_{u}$ and $F^{3} \Pi_{u}$ state excitations}

In Figs. 8 and 9, we show representative DCSs for excitations to the $3 p \pi_{u} G^{3} \Pi_{u}$ and $3 s \sigma_{g} F^{3} \Pi_{u}$ Rydberg states of $\mathrm{N}_{2}$ at $E_{0}=17.5$ and $50 \mathrm{eV}$. At $17.5 \mathrm{eV}$, these excitations display DCSs that are typical of forbidden excitations, with a downturn at small $\theta$, and angular distributions that are similar, as would be expected for transitions to triplet Rydberg states of the same symmetry. At large $\theta$, the $F^{3} \Pi_{u}$ state DCSs show a different angular behavior, possibly indicating that these states may be coupled differently to neighboring states. However, some caution must be applied to the assessment of the $G^{3} \Pi_{u}$ state and $F^{3} \Pi_{u}$ state DCSs. As pointed out in Sec. II B, while several of their levels are resolved experimentally, the use of theoretical (RKR) FC factors to estimate the remaining intensity is an imperfect, albeit necessary, process. We note that in this case we are considering effectively $100 \%$ of the $G^{3} \Pi_{u}$ and $F^{3} \Pi_{u}$ states in our analysis, since almost all of their intensity lies in the energy-loss range of the present work.

Again, the DCSs of Trajmar et al. [19] were taken at $E_{0}$ $=40$ and $60 \mathrm{eV}$ with an average of their two data sets used here for comparison. At $E_{0}=50 \mathrm{eV}$, their DCSs (with $25 \%$ uncertainty) are somewhat lower than ours at forward angles, but are in good agreement at intermediate angles. Marked deviations between our results and theirs are observed for $\theta>70^{\circ}$. Whereas both sets of DCSs show an upturn, the DCSs of Trajmar et al. [19] are considerably larger than ours in this region. Considering the incorrect assignments in the work of Chutjian et al. [26] from which the work in [19] is based, it is indeed surprising that there is any degree of agreement with the DCSs of this work. In the case of the $G^{3} \Pi_{u}$ state, the DCS of [26] includes the correct $G(0)$ level, but includes the $b(6)$ level instead of $G(1)$, and no higher levels. Further, Chutjian et al. [26] had to include the unidentified levels $M 1, M 2$, and $M 3$ in the unfolding of their experimental spectra to obtain satisfactory fits. In fact, these levels correspond to $G(1), G(2)$, and $G(3)$. The smaller than expected discrepancy between the two $G^{3} \Pi_{u}$ state DCSs can perhaps be explained by the large FC factor used by Chutjian et al. for $G(0)$, together with some compensation for the missing $G(2)$ and $G(3)$ contributions by the inclusion of $b(6)$. We note here, in passing that, due to quantuminterference effects, the behavior of the dipole-allowed excitation to $b(6)$ mimics that of a dipole-forbidden transition, with a relative increase in intensity at high scattering angles. In the case of the $F^{3} \Pi_{u}$ state, the DCSs of Chutjian et al. [26] are based on an incorrectly numbered vibrational series, with their $F(0)$ coinciding with $b(3)$ and their $F(1)$ actually $F(0)$. Clearly, this will have led to an incorrect application of FC factors and subsequent problems with their DCSs. Consequently, we recommend our DCSs for the $G^{3} \Pi_{u}$ and $F^{3} \Pi_{u}$ states.

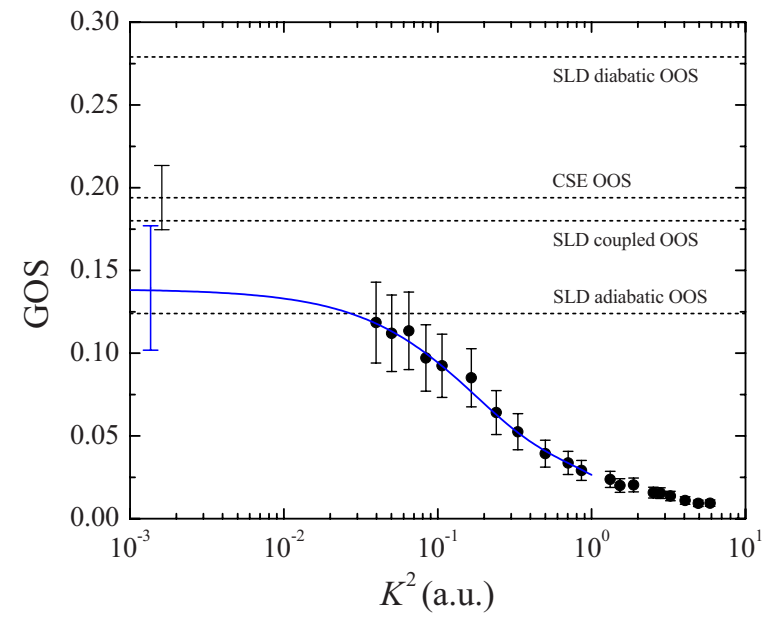

FIG. 10. (Color online) GOSs for the $b^{1} \Pi_{u}$ state derived from the present $(\mathbf{O}) 100 \mathrm{eV}$ DCS results and adjusted for unmeasured $v^{\prime}$ levels (see Table IV). A Vriens polynomial extrapolation of the GOSs is shown (solid line), based on the derived GOSs, with the uncertainty indicated. The dashed horizontal lines indicate the Stahel et al. (adiabatic, diabatic, and coupled) [8] and CSE [61] OOS values. The CSE OOS error bar assumes $10 \%$ uncertainty in order to illustrate the consistency between data sets. See text for additional discussion.

\section{B. Excitation optical oscillator strengths}

As a physical consistency check, optical oscillator strengths (OOSs) were estimated based on present DCSs for optically allowed transitions and compared with available OOS values. DCSs at $E_{0}=100 \mathrm{eV}$ were converted into generalized oscillator strengths according to the following relation:

$$
\left[\operatorname{GOS}\left(K^{2}\right)\right]=\frac{E}{2} K^{2}\left(\frac{E_{0}}{E_{R}}\right)^{1 / 2}(\mathrm{DCS}),
$$

where $K^{2}, E, E_{0}, E_{R}$, and the DCSs are in a.u. These GOSs were then extrapolated to $K^{2} \rightarrow 0$, giving the associated OOSs, using the (well-established) polynomial extrapolation method developed by Vriens [60]. Note that the GOSs were first corrected to account for vibrational levels in the measured DCSs that were not within the measured energy-loss range. The GOSs were scaled by factors given by the sum of renormalized REPs for levels within our measured energyloss range [see the P (renorm) REPs in Table I]. So, for example, the $b{ }^{1} \Pi_{u}$ state GOSs, shown in Fig. 10, were divided by 0.973 . The results of this procedure, given in Table IV, are compared with OOS values from Stahel et al. [8] and Lewis [61].

The diabatic and adiabatic OOSs of Stahel et al. listed in Table IV were computed from their published data (see their Table XV) using the formula

$$
(\mathrm{OOS})=\frac{2}{3} \frac{g_{j}}{g_{i}}\left|M_{i j}\right|^{2} E
$$

for a transition from a lower state $i$ to an upper state $j$, where $M_{i j}$ is the appropriate transition moment, and the statisticalweight ratio $g_{i} / g_{j}=1$, for ${ }^{1} \Sigma \rightarrow{ }^{1} \sum$ transitions, or $g_{i} / g_{j}=2$, for 
TABLE IV. Optical oscillator strength (OOS) values for the electron-impact excitation of the $X^{1} \Sigma_{g}^{+}\left(v^{\prime \prime}\right.$ $=0) \rightarrow b{ }^{1} \Pi_{u}, c_{3}{ }^{1} \Pi_{u}, o_{3}{ }^{1} \Pi_{u}, b^{\prime}{ }^{1} \Sigma_{u}^{+}$, and $c_{4}^{\prime}{ }^{1} \Sigma_{u}^{+}$transitions in $\mathrm{N}_{2}$. The present values (P) were obtained from GOSs derived from the present $E_{0}=100 \mathrm{eV}$ DCSs. See text for details regarding the limitations of the incident electron energy and vibrational coverage via the energy-loss range employed in the present work (Sec. III B). For each electronic state, scaling factors, equivalent to the renormalized REPs [i.e., P (renorm)] in Table I, were applied to the GOSs to account for $v^{\prime}$ levels outside the measured energy-loss range. See text for additional discussion, particularly for the $b^{\prime}{ }^{1} \Sigma_{u}^{+}$and $c_{4}^{\prime}{ }^{1} \Sigma_{u}^{+}$states.

\begin{tabular}{lcccccc}
\hline \hline State & $\begin{array}{c}\text { Scaling } \\
\text { factor }\end{array}$ & $\begin{array}{c}\text { OOS } \\
(\mathrm{P})\end{array}$ & $\begin{array}{c}\text { OOS } \\
\text { diabatic }) \\
{[8]}\end{array}$ & $\begin{array}{c}\text { OOS } \\
\text { (adiabatic) } \\
{[8]}\end{array}$ & $\begin{array}{c}\text { OOS } \\
\text { (coupled) } \\
{[8]}\end{array}$ & $\begin{array}{c}\text { OOS } \\
\text { (CSE) } \\
{[45]}\end{array}$ \\
\hline$b{ }^{1} \Pi_{u}$ & 0.973 & $0.139 \pm 0.038$ & 0.279 & 0.124 & 0.180 & 0.194 \\
$c_{3}{ }^{1} \Pi_{u}$ & 1.000 & $0.087 \pm 0.024$ & 0.033 & 0.141 & 0.080 & 0.102 \\
$o_{3}{ }^{1} \Pi_{u}$ & 0.912 & $0.041 \pm 0.017$ & 0.009 & 0.061 & 0.061 & 0.044 \\
$b^{\prime}{ }^{1} \Sigma_{u}^{+}$ & 0.081 & $0.463 \pm 0.144$ & 0.221 & 0.209 & 0.194 & 0.239 \\
$c_{4}{ }^{1} \Sigma_{u}^{+}$ & 0.825 & $0.092 \pm 0.030$ & 0.113 & 0.139 & 0.172 & 0.188 \\
Summed & & $0.822 \pm 0.154$ & 0.655 & 0.674 & 0.687 & 0.767 \\
\hline \hline
\end{tabular}

${ }^{1} \Sigma \rightarrow{ }^{1} \Pi$ transitions. The coupled OOSs of Stahel et al. were obtained using the $M_{\text {calc }}^{2}$ values in their Tables III and IV, together with the scaling factors given in the caption to their Fig. 4 [8].

Differences between our approximated OOS values and other data sets is almost certainly due to our extrapolation procedure and should not be misconstrued as an underlying problem with our energy-loss and DCS data. At $100 \mathrm{eV}$, the present data are not sufficiently close to $K^{2}=0$ to allow accurate extrapolation of the GOSs to their corresponding OOSs. As seen in the example shown in Fig. 10, the present extrapolations are poorly constrained by the available data (i.e., not in the Born region). However, as stated earlier, this procedure was intended as a consistency check and not as a means to derive, improve, or determine accurate OOSs. All the present GOSs were found to be consistent with available OOSs and thereby support the validity of the underlying DCSs even though they were not able to constrain accurate OOS predictions.

The present OOS values would be significantly and primarily improved by extending the measurements to larger incident electron energies $(>500 \mathrm{eV}$-i.e., the Born region) and thereby further constraining the extrapolations to $K^{2}=0$. The results would also be improved by an extension of the unfolded energy-loss range, particularly allowing for the inclusion of significantly more levels of the $b^{\prime}{ }^{1} \Sigma_{u}^{+}$state (the scaling factor used to account for unmeasured $v^{\prime}$ levels was $\left.0.0805^{-1}\right)$. However, as the energy-loss coverage approaches the ionization limit, the number of contributing Rydberg levels increases significantly. This causes difficulty in resolving and unfolding spectral features, particularly when the primary task is obtaining DCSs over many angles, which is accomplished by increasing signal intensities to reasonable levels. So, for example, the significant difference between our $c_{4}^{\prime}(3)$ "REP" and those of Stahel et al. (coupled) [8] may be due to uncertainties compounded in resolving this highlying level from the $b(13)$ level. [Note that Walter et al. [62] observed strong perturbations in the highly mixed rovibronic levels within the $c_{4}^{\prime}(3) / c_{3}(3) / b^{\prime}(10)$ complex.] We further note that a larger REP for the $c_{4}^{\prime}(3)$ level should also increase the present $c_{4}^{\prime}{ }^{1} \Sigma_{u}^{+}$state DCS slightly and, more importantly, increase the OOS towards better agreement with the CSE results [61]. The reader is referred to $[63,64]$ and references therein for a more comprehensive survey of OOS values for $\mathrm{N}_{2}$.

\section{CONCLUSIONS}

Using a high-resolution electron spectrometer, as well as accurate energy levels for the vibronic levels of the $\mathrm{N}_{2}$ molecule in the $12-13.82 \mathrm{eV}$ excitation energy-loss range, we have been able to unfold much of the molecular spectrum and obtain improved DCSs for the excitations to the corresponding electronic states. The present work obtains DCSs for excitations to the heavily mixed Rydberg and valence states from the ground state, which attempts to account for the effects of state mixing. Further, it is the first attempt to provide these over a large range of $E_{0}$ and $\theta$. This contrasts with the earlier work of Trajmar et al. [19], which was done at two rather closely spaced $E_{0}$ values of 40 and $60 \mathrm{eV}$, and used an analysis based on available FC factors. The following results were particularly noteworthy.

(a) The present DCSs at $E_{0}=50 \mathrm{eV}$ compared surprisingly well with those of [19], which were taken from the averaged DCSs of their $E_{0}=40$ and $60 \mathrm{eV}$ data sets. This suggests that the least-squares fitting procedures employed by [26] in the spectral unfolding were unexpectedly insensitive to the FC factor basis they used, perhaps somewhat understandable in cases where the strongest features in a sequence are almost fully resolved.

(b) Similar to the observations of Trajmar and co-workers $[19,46]$, we were unable to observe any significant cross section for excitation to the $D^{3} \Sigma_{u}^{+}$metastable state.

(c) Significant scattering-condition-dependent deviations of REPs from FC factors were found for vibrational features in the $b^{1} \Pi_{u}-X^{1} \Sigma_{g}^{+}$and $c_{3}{ }^{1} \Pi_{u}-X^{1} \Sigma_{g}^{+}$systems.

(d) A very interesting cusp was observed for the DCS of the $a^{\prime \prime}{ }^{1} \Sigma_{g}^{+}$state at $\theta \approx 20^{\circ}$. This cusp begins at $E_{0} \approx 20 \mathrm{eV}$ and persists to (at least) $100 \mathrm{eV}$. This is the first excitation cusp observed for molecules and we hypothesize that it is a 
result of channel coupling since the ground $X^{1} \Sigma_{g}^{+}$and excited $a^{\prime \prime}{ }^{1} \Sigma_{g}^{+}$states have the same ${ }^{1} \Sigma_{g}^{+}$symmetry. A closecoupling calculation could reveal such an effect.

\section{ACKNOWLEDGMENTS}

This work was performed at the California State University, Fullerton (CSUF), Australian National University (ANU), and at the Jet Propulsion Laboratory (JPL), California Institute of Technology (Caltech), under a contract with the National Aeronautics and Space Administration (NASA). We gratefully acknowledge financial support through the National Science Foundation, under Grant No. NSF-PHY-RUI0096808, and NASA's Outer Planets Research program. The work at the ANU was supported by the Australian Research Council Discovery Program, through Grant No. DP0558962. This research was performed while C.P.M. received additional support from NASA at JPL. Valuable communications with Dr. K. Bartschat (Drake University, Iowa) regarding channel-coupling effects are greatly appreciated.
[1] M. A. Khakoo, P. V. Johnson, I. Ozkay, P. Yan, S. Trajmar, and I. Kanik, Phys. Rev. A 71, 062703 (2005).

[2] R. F. da Costa and M. A. P. Lima, Int. J. Quantum Chem. 106, 2664 (2006).

[3] R. F. da Costa and M. A. P. Lima, Phys. Rev. A 75, 022705 (2007).

[4] M. Tashiro and K. Morokuma, Phys. Rev. A 75, 012720 (2007).

[5] S. J. Buckman (unpublished).

[6] H. Lefebvre-Brion and R. W. Field, The Spectra and Dynamics of Diatomic Molecules (Elsevier, San Diego, 2004).

[7] D. Spelsberg and W. Meyer, J. Chem. Phys. 115, 6438 (2001).

[8] D. Stahel, M. Leoni, and K. Dressler, J. Chem. Phys. 79, 2541 (1983).

[9] J. P. Sprengers, E. Reinhold, W. Ubachs, K. G. H. Baldwin, and B. R. Lewis, J. Chem. Phys. 123, 144315 (2005).

[10] B. R. Lewis, S. T. Gibson, W. Zhang, H. Lefebvre-Brion, and J. M. Robbe, J. Chem. Phys. 122, 144302 (2005).

[11] B. R. Lewis, J. P. England, S. T. Gibson, M. J. Brunger, and M. Allan, Phys. Rev. A 63, 022707 (2001).

[12] M. A. Khakoo, S. Wang, R. Laher, P. V. Johnson, C. P. Malone, and I. Kanik, J. Phys. B 40, F167 (2007).

[13] D. G. Wilden, P. J. Hicks, and J. Comer, J. Phys. B 12, 1579 (1979).

[14] Y. Itikawa, M. Hayaski, A. Ichimura, K. Onda, K. Sakimoto, K. Takayanagi, M. Nakamura, H. Nishimura, and T. Takayanagi, J. Phys. Chem. Ref. Data 15, 985 (1986).

[15] Y. Itikawa, J. Phys. Chem. Ref. Data 35, 31 (2006).

[16] A. Lofthus and P. H. Krupenie, J. Phys. Chem. Ref. Data 6, 113 (1977).

[17] E. N. Lassettre, A. Skerbele, and V. D. Meyer, J. Chem. Phys. 45, 3214 (1966).

[18] D. C. Cartwright, A. Chutjian, S. Trajmar, and W. Williams, Phys. Rev. A 16, 1013 (1977).

[19] S. Trajmar, D. F. Register, and A. Chutjian, Phys. Rep. 97, 219 (1983).

[20] M. J. Brunger and P. J. O. Teubner, Phys. Rev. A 41, 1413 (1990).

[21] M. Furlan, M. J. Hubinfranskin, J. Delwiche, and J. E. Collin, J. Phys. B 23, 3023 (1990).

[22] S. Trajmar, J. K. Rice, and A. Kuppermann, in Advances in Chemical Physics, edited by I. Prigogine and S. A. Rice (Interscience, Toronto, 1970), Vol. XVIII, p. 15.

[23] M. Zubek and G. C. King, J. Phys. B 27, 2613 (1994).

[24] P. K. Carroll and C. P. Collins, Can. J. Phys. 47, 563 (1969).
[25] K. Dressler, Can. J. Phys. 47, 547 (1969).

[26] A. Chutjian, D. C. Cartwright, and S. Trajmar, Phys. Rev. A 16, 1052 (1977).

[27] J. Geiger and B. Schröder, J. Chem. Phys. 50, 7 (1969).

[28] G. Joyez, R. I. Hall, J. Reinhardt, and J. Mazeau, J. Electron Spectrosc. Relat. Phenom. 2, 183 (1973).

[29] T. J. Whang, G. X. Zhao, W. C. Stwalley, and C. Y. R. Wu, J. Quant. Spectrosc. Radiat. Transf. 55, 335 (1996).

[30] J. M. Ratliff, G. K. James, S. Trajmar, J. M. Ajello, and D. E. Shemansky, J. Geophys. Res. 96, 17559 (1991).

[31] P. K. Carroll, C. P. Collins, and K. Yoshino, J. Phys. B 3, L127 (1970).

[32] L. Mu-Tao and V. McKoy, Phys. Rev. A 28, 697 (1983).

[33] J. P. Sprengers, A. Johansson, A. L'Huillier, C.-G. Wahlström, B. R. Lewis, and W. Ubachs, Chem. Phys. Lett. 389, 348 (2004).

[34] J. P. Sprengers, W. Ubachs, and K. G. H. Baldwin, J. Chem. Phys. 122, 144301 (2005).

[35] J. P. Sprengers, W. Ubachs, A. Johansson, A. L'Huillier, C.-G. Wahlström, R. Lang, B. R. Lewis, and S. T. Gibson, J. Chem. Phys. 120, 8973 (2004).

[36] V. E. Haverd, B. R. Lewis, S. T. Gibson, and G. Stark, J. Chem. Phys. 123, 214304 (2005).

[37] G. Stark, K. P. Huber, K. Yoshino, P. L. Smith, and K. Ito, J. Chem. Phys. 123, 214303 (2005).

[38] A. B. van der Kamp, P. C. Cosby, and W. J. van der Zande, Chem. Phys. 184, 319 (1994).

[39] H. Lefebvre-Brion, J. Chem. Phys. 122, 144315 (2005).

[40] E. F. McCormack, S. T. Pratt, J. L. Dehmer, and P. M. Dehmer, Phys. Rev. A 42, 5445 (1990).

[41] M. A. Khakoo, C. E. Beckmann, S. Trajmar, and G. Csanak, J. Phys. B 27, 3159 (1994).

[42] M. Hughes, K. E. James, J. G. Childers, and M. A. Khakoo, Meas. Sci. Technol. 14, 841 (2003).

[43] L. R. LeClair and S. Trajmar, J. Phys. B 29, 5543 (1996).

[44] E. Schow, K. Hazlett, J. G. Childers, C. Medina, G. Vitug, I. Bray, D. V. Fursa, and M. A. Khakoo, Phys. Rev. A 72, 062717 (2005).

[45] B. R. Lewis (unpublished). Note that the state energies were determined by a review of all available experimental data and an overall CSE assessment of the ${ }^{1} \Pi_{u},{ }^{1} \Sigma_{u}^{+}$, and ${ }^{3} \Pi_{u}$ electronic states. Of most relevance here are the new assignments for vibrational levels of the triplet states in Table I.

[46] S. Trajmar (personal communication).

[47] J. C. Nickel, P. W. Zetner, G. Shen, and S. Trajmar, J. Phys. E 
22, 730 (1989).

[48] D. C. Cartwright, Phys. Rev. A 2, 1331 (1970).

[49] D. C. Cartwright, Phys. Rev. A 5, 1974 (1972).

[50] S. K. Srivastava, A. Chutjian, and S. Trajmar, J. Chem. Phys. 64, 1340 (1976).

[51] T. W. Shyn and G. R. Carignan, Phys. Rev. A 22, 923 (1980).

[52] J. C. Nickel, C. Mott, I. Kanik, and D. C. McCollum, J. Phys. B 21, 1867 (1988).

[53] M. Gote and H. Ehrhardt, J. Phys. B 28, 3957 (1995).

[54] M. J. Brunger and S. J. Buckman, Phys. Rep. 357, 215 (2002).

[55] F. R. Gilmore, R. R. Laher, and P. J. Espy, J. Phys. Chem. Ref. Data 21, 1005 (1992).

[56] S. Y. Wang, P. V. Johnson, C. P. Malone, I. Kanik, and M. A. Khakoo, Phys. Rev. A 73, 034702 (2006).

[57] L. Campbell, M. J. Brunger, A. M. Nolan, L. J. Kelly, A. B. Wedding, J. Harrison, P. J. O. Teubner, D. C. Cartwright, and B. McLaughlin, J. Phys. B 34, 1185 (2001).

[58] P. V. Johnson, C. P. Malone, I. Kanik, K. Tran, and M. A.
Khakoo, J. Geophys. Res. 110, A11311 (2005).

[59] A. W. Kam and F. M. Pipkin, Phys. Rev. A 43, 3279 (1991).

[60] L. Vriens, Phys. Rev. 160, 100 (1967).

[61] B. R. Lewis (unpublished). Note that optical oscillator strengths were determined by fitting a semiempirical CSE model to an experimental oscillator-strength database, primarily from the work of Stark et al. [37] (including unpublished data). Also see Ref. [45].

[62] C. W. Walter, P. C. Cosby, and H. Helm, J. Chem. Phys. 112, 4621 (2000).

[63] W. F. Chan, G. Cooper, R. N. S. Sodhi, and C. E. Brion, Chem. Phys. 170, 81 (1993).

[64] X. M. Liu and D. E. Shemansky, Astrophys. J. 645, 1560 (2006).

[65] P. Hammond, G. C. King, J. Jureta, and F. H. Read, J. Phys. B 20, 4255 (1987).

[66] H. Partridge (personal communication). 